\title{
29. GEOCHEMISTRY OF SEDIMENTS AT SITES 579, 580, AND 581, DEEP SEA DRILLING PROJECT LEG 86, WESTERN NORTH PACIFIC ${ }^{1}$
}

\author{
G. Ross Heath, Richard B. Kovar, and Carlos Lopez, Oregon State University²
}

\begin{abstract}
The elemental composition of sediments from Sites $579\left(38^{\circ} 38^{\prime} \mathrm{N}, 153^{\circ} 50^{\prime} \mathrm{E}\right)$ and $580\left(41^{\circ} 37^{\prime} \mathrm{N}, 153^{\circ} 59^{\prime} \mathrm{E}\right)$ is dominated by contributions from terrigenous detritus (including numerous thin ash beds) and opal-rich biogenic debris, with the terrigenous component increasing in dominance in the youngest sediments as the flux of Pleistocene eolian debris associated with Northern Hemisphere glaciation has increased. Diagenesis related to redox-sensitive reactions in near-surface sediments has had a marked impact on the distributions of $\mathrm{Mn}$ and $\mathrm{S}$.

At Site $581\left(43^{\circ} 56^{\prime} \mathrm{N}, 159^{\circ} 48^{\prime} \mathrm{E}\right)$, the reduced biosiliceous clays corresponding to the sections at Sites 579 and 580 are underlain by a normal oxidized North Pacific "red clay" sequence. As at other North Pacific sites, the concentrations of $\mathrm{Mn}$ and $\mathrm{Fe}$ (as oxyhydroxides), $\mathrm{Ba}$ (as barite), and $\mathrm{P}$ (as fish debris) vary inversely with the accumulation rates of the clays. The cherts underlying the clays at Site 581 are noteworthy for their high P contents (comparable to values for biosiliceous clays) and high $\mathrm{Fe}$ and $\mathrm{Mn}$ relative to $\mathrm{Ti}$ and $\mathrm{Al}$, suggestive of derivation from well-oxidized pelagic sediments.
\end{abstract}

\section{INTRODUCTION}

\section{Setting}

During Deep Sea Drilling Project (DSDP) Leg 86, holes were drilled in pure calcareous ooze (Site 577), pelagic "red" clay (Site 576), and carbonate-free pelagic clay with variable contents of biogenic silica and volcanic ash (Sites 578-581). This chapter focuses on the elemental composition of sediments from Sites 579 $\left(38^{\circ} 38^{\prime} \mathrm{N}, 153^{\circ} 50^{\prime} \mathrm{E}\right), 580\left(41^{\circ} 37^{\prime} \mathrm{N}, 153^{\circ} 59^{\prime} \mathrm{E}\right)$, and $581\left(43^{\circ} 56^{\prime} \mathrm{N}, 159^{\circ} 48^{\prime} \mathrm{E}\right)$ (Fig. 1) in order to assess the roles of temporally varying biogenic and ash inputs and of in situ redox conditions in establishing the geochemical character of such deposits. The soft sediments at Sites 579 and 580 were cored continuously by hydraulic piston corer. The more indurated Site 581 sediments were recovered by conventional wire-line rotary coring. Because the upper section (0-181.5 m sub-bottom) of Hole 581 was not sampled, we report only on deeper portions of the section at this site.

The descriptions of the sediments at Sites 579 and 580 (see Site 579 and 580 chapters, this volume) show them to be uniform gray and greenish gray siliceous clay and siliceous ooze that accumulated at rates of about 35 and $50 \mathrm{~m} / \mathrm{m} . \mathrm{y}$. , respectively, interrupted by 61 (Site 579 ) and 89 (Site 580) identifiable ash beds and numerous dark greenish gray indurated layers that may be altered basic ash layers. Our samples, taken approximately 1.5 $\mathrm{m}$ apart, were placed so as to avoid both ashes and indurated layers. The color of the sediment and the presence of fine pyrite grains point to strongly reduced conditions $\left(\mathrm{Fe}^{3+}\right.$ and $\mathrm{SO}_{4}{ }^{2-}$ reduction) throughout both sections.

\footnotetext{
${ }^{1}$ Heath, G. R., Burckle, L. H., et al., Init. Repts. DSDP, 86: Washington (U.S. Govt. Printing Office).

2 Address: (Heath, present address) College of Ocean and Fishery Sciences, University of Washington, Seattle, WA 98195; (Kovar, Lopez) College of Oceanography, Oregon State University, Corvallis, OR 97331.
}

The section from 0 to $224 \mathrm{~m}$ at Site 581 (0-9 m.y. ago) is assumed to resemble the sections at Sites 579 and 580, although only the interval below $181 \mathrm{~m}$ (with an accumulation rate decreasing downcore from $\sim 12$ to $\sim 4 \mathrm{~m} /$ m.y.) was cored continuously. From 224 to $245 \mathrm{~m}$ ( 9 to $\sim 14 \mathrm{~m} / \mathrm{m} . \mathrm{y}$. ago), the sediments are brown and light brown pelagic clays (that accumulated at about $4 \mathrm{~m} /$ m.y.), with small but variable concentrations of biogenic silica that decrease downcore and are rare at the base of the interval. The deepest clays $(245-276 \mathrm{~m})$ are very dark brown "slick" pelagic deposits typical of early to middle Tertiary deposits from vast areas of the North Pacific. Below $276 \mathrm{~m}$, only small fragments of porcellaneous chert were recovered. From the behavior of the drill, the chert is believed to form thin beds in pelagic clay. The intense circulation of seawater required to keep chert fragments away from the bit washed away all the soft sediment, however.

\section{Methodology}

Samples were freeze-dried, disaggregated by shaking with methacrylate spheres in polycarbonate sample tubes, pressed into pellets, and analyzed by X-ray fluorescence using a Phillips PW-1600 simultaneous spectrometer. Raw counts for each element were ratioed to U.S. Geological Survey (USGS) standard SY3, which was run before and after each sample, and then converted to concentrations using a fundamental parameters program (Criss et al., 1978). Analyses of standard rocks and sediments indicate no systematic errors for the elements discussed. Analytical precision (counting statistics) is better than $2 \%$ for the elements reported, but sample to sample variability at the same level in a core is closer to $5 \%$ of the reported concentrations.

In order to determine the number of components in the Leg 86 sediments, we analyzed the elemental data using Q-mode factor analysis (Klovan and Imbrie, 1971). The empirical orthogonal functions (factors) generated 


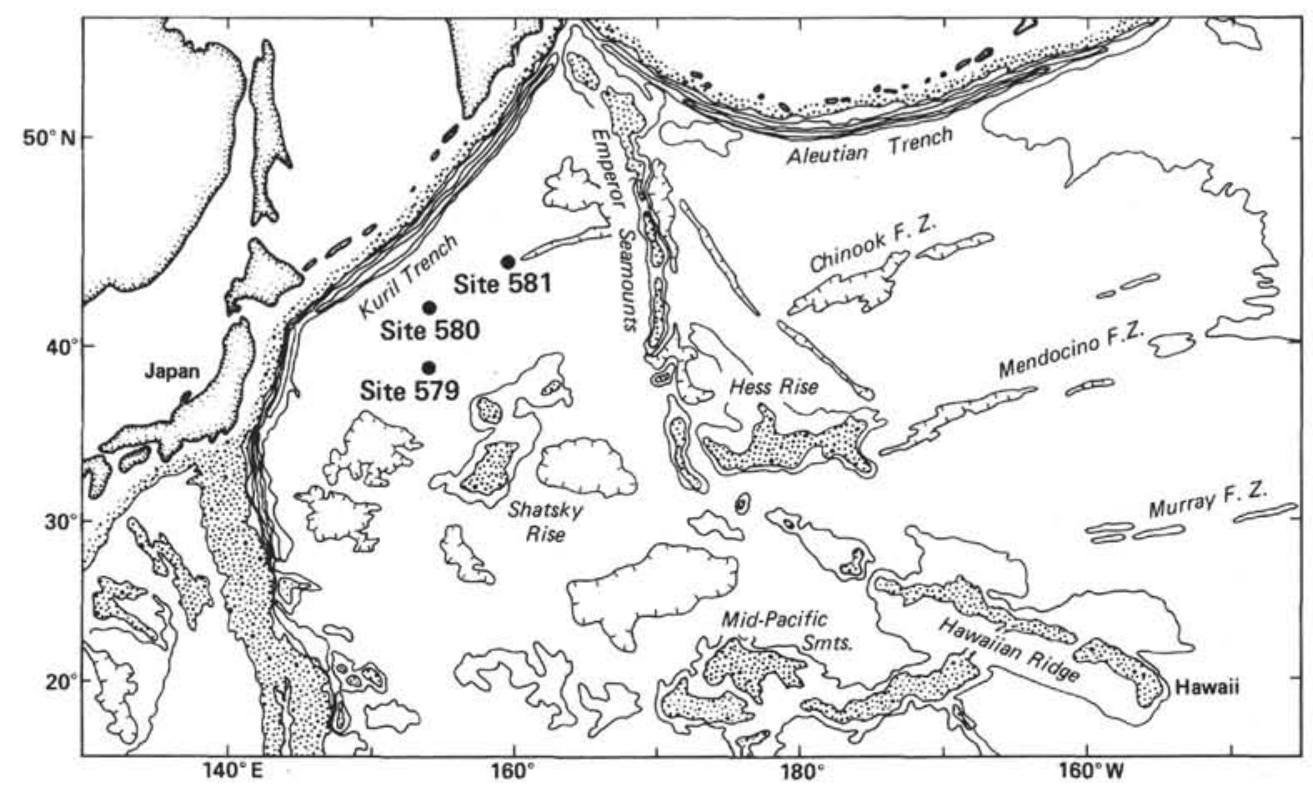

Figure 1. Location of Sites 579, 580, and 581 relative to major physiographic features of the western North Pacific (after Chase, 1975). Areas shallower than $4 \mathrm{~km}$ stippled; $5-\mathrm{km}$ contour plain; 6-km contour hachured.

by this technique distribute the variance in the raw data as equally as possible between a subjectively selected number of "factors." We chose three factors in all of our analyses because this number accounted for $97 \%$ or more of the variance in the data sets.

\section{RESULTS}

The major-element analytical results for Sites 579, 580, and 581 are summarized in Tables 1 to 3 . Figures 2 to 4 show downhole plots of elemental abundances that illustrate the major compositional variations at the three sites.

Titanium, which is typical of elements supplied in detritus, shows similar distributions in the sections at Sites 579 and 580 (Fig. 2). For the past 1 m.y., values have been relatively high $(0.36$ and $0.35 \%$, respectively), with standard deviations of 8 to $10 \%$. Concentrations decrease downhole by about a third in the deposits older than 2 m.y., apparently due to dilution of the terrigenous component by biogenic opal. At the base of Hole 579 , which is older than the base of Hole 580, Ti and several other elements (Fig. 2) increase slightly.

The profile at Site 581, which is older than the two preceding sites, shows higher $\mathrm{Ti}$ values prior to $5 \mathrm{~m}$.y. ago (Fig. 4). The concentration increases downhole in steps, with an increase of approximately $0.05 \%$ at 9 m.y. $(225 \mathrm{~m})$ and a similar $0.05 \%$ increase at about $14 \mathrm{~m} . \mathrm{y}$. $(245 \mathrm{~m})$. These increases correspond to the downhole changes in lithology from gray green siliceous clay to light brown pelagic clay to dark brown pelagic clay, respectively (see Site 581 chapter, this volume).

Below $270 \mathrm{~m}$, only chert was recovered from the sedimentary section at Site 581. Ti values for the cherts are three to five times lower than in the overlying sediments (due to dilution by silica), but decrease downhole suggesting a reversal of the lithologic trend observed from 180 to $270 \mathrm{~m}$ (Fig. 4). Because the time duration of the interval represented by the chert section is unknown, we can only infer that the low $\mathrm{Ti}$ values at the base of the section are indicative of biogenic dilution of terrigenous debris at the time when the site lay beneath the equatorial zone of high biological productivity.

Other elements with distributions similar to $\mathrm{Ti}$, particularly at Sites 579 and 580 , are $\mathrm{A} 1, \mathrm{Mg}, \mathrm{K}$, and $\mathrm{Fe}$ (Fig. 2). At Site 581, Fe diverges from $\mathrm{Ti}$ in the deeper cherts, reaching up to $50 \%$ of the detrital values below $300 \mathrm{~m}$ (Fig. 4). One possible explanation for such a divergence would be the deposition of excess hydrothermal $\mathrm{Fe}$ beneath the equator (Leinen and Stakes, 1979).

$\mathrm{Si}$, much of which is supplied as biogenic opal, varies almost inversely with the terrigenous elements. Shortterm variability is again about $10 \%$, with the more northerly (and more productive) Site 580 showing more variability than Site 579 , particularly during the past $1 \mathrm{~m} . \mathrm{y}$. (Fig. 2).

At Site 581, the light brown (224-245 $\mathrm{m}$ ) and gray green (above $224 \mathrm{~m}$ ) sections and the chert samples (below $276 \mathrm{~m}$ ) are all silica rich (Fig. 4). In fact, the chert values $(32.8 \pm 1.3 \%)$ are indistinguishable from those for the light brown pelagic clays $(31.8 \pm 1.4 \%)$. In contrast, the dark brown pelagic clays $(245-276 \mathrm{~m})$ are silica depleted, with a mean value of only $24.2 \%$ (and a standard deviation of $2.4 \%$ ).

$\mathrm{Mn}$ and $\mathrm{S}$ are both redox-sensitive elements. $\mathrm{Mn}$ is reduced from the insoluble +4 to soluble +2 state under mildly reducing (suboxic) conditions (Froelich et al., 1979), whereas $\mathrm{S}$ is reduced from +6 to $-2\left(\mathrm{SO}_{4}{ }^{2-}\right.$ to $\left.\mathrm{S}^{2-}\right)$ only when $\mathrm{O}_{2}, \mathrm{NO}_{3}{ }^{-}, \mathrm{Mn}^{4+}$, and $\mathrm{Fe}^{3+}$ have been consumed.

At Site 579, virtually all the oxyhydroxide Mn has been reduced and has diffused out of the sediments (Fig. 3). Spikes near the base of the section and near the surface are the only indicators of more oxidized conditions. In contrast, the more rapidly deposited sediments at Site 
Table 1. Elemental composition (wt.\%) of Site 579 sediment samples.

\begin{tabular}{|c|c|c|c|c|c|c|c|c|c|c|c|c|c|c|c|}
\hline $\begin{array}{c}\text { OSU } \\
\text { Lab } \\
\text { Sample }\end{array}$ & ปั & 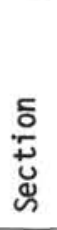 & 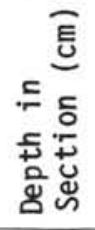 & $\begin{array}{l}=\bar{E} \\
\text { 틍 } \\
\text { 흥응 }\end{array}$ & Mn & $\mathrm{Fe}$ & Si & Al & K & $\mathrm{Ca}$ & $\mathrm{Mg}$ & $\mathrm{Ti}$ & $P$ & $\mathrm{Ba}$ & $S$ \\
\hline 8 & 1 & 1 & & נדיט & 38 & 1 & 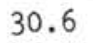 & & 02 & & 52 & 0 & 67 & 4 & .084 \\
\hline DP10809 & 1 & 2 & & 列 & .01 & 5.19 & 31.7 & 8.01 & 2.26 & & & & & & \\
\hline DP10810 & 1 & 3 & 0.35 & 3.35 & .03 & 2.92 & 31.9 & 6.75 & 19 & & 1.09 & & & & .139 \\
\hline DP10811 & 1 & 4 & 0.35 & 4.85 & 0 & & & & 2.26 & & & & 0.052 & & \\
\hline DP10812 & 1 & 5 & 0.35 & 6.35 & 0.07 & 5.36 & & & 2.03 & & & & & & \\
\hline DP10813 & 1 & 6 & 0.35 & 7.85 & 0.04 & 3.71 & & & 2.08 & & & & 0. & & \\
\hline DP10814 & 2 & 1 & 0.71 & & 0.02 & 4.27 & & & 2.23 & & & & 0.051 & & \\
\hline DP10815 & 2 & 2 & 0.71 & 10.61 & 0.02 & 3.99 & & & & & & & & & \\
\hline 0816 & 2 & 3 & 0.71 & & & & & & & & & & & & \\
\hline 0817 & 2 & 4 & 0.71 & & & & & & & & & & & & \\
\hline DP 10818 & 2 & 5 & 0.71 & & 0.02 & 3. & 30.0 & 7. & 2.14 & & & & & & \\
\hline DP10819 & 2 & 6 & 0.71 & & 0.01 & & & & 2.23 & & 1. & & & & \\
\hline DP10820 & 1 & 1 & 1.15 & & 0.03 & & & & & & & & & & \\
\hline DP1 & 1 & 2 & 1.15 & & 0 & & & & & & & & & & \\
\hline DP10 & 1 & 3 & 1.15 & & 0 & & & & & & & & & & \\
\hline 23 & 1 & 4 & 1.15 & & 0.03 & & & & 2.46 & & & & & & \\
\hline 24 & 1 & 5 & 1.15 & & 0.02 & 4. & & 7. & 2.03 & & & & & & \\
\hline 5 & 1 & 5 & 1.15 & & 0.02 & 3. & & 8. & 2.18 & & & & & & 0.107 \\
\hline 26 & 1 & 7 & 0.13 & & 0.01 & & & 7. & 2.11 & & & & & & \\
\hline 0827 & 2 & 1 & 0.59 & 9 & 0.03 & & & & & & & & & & .127 \\
\hline DP10828 & 2 & 2 & 0.59 & & & & & & & & & & & & .094 \\
\hline DP10829 & 2 & 3 & 0.59 & & 0.01 & & & & 2.22 & & & & & & \\
\hline 30 & 2 & 4 & 0.51 & & 0,01 & & & & 1 & & & & & & \\
\hline DP1 & 2 & 5 & 0.59 & & 2 & & 31 & & 2 & & & & & & \\
\hline 32 & 2 & 6 & 0.59 & & 2 & & & & 2. & & & & & & \\
\hline 3 & 2 & 7 & 0.19 & & 0.01 & & & & 2.20 & & & & & & 46 \\
\hline 34 & 3 & 1 & 0.65 & & 0.03 & 4. & & & 2.47 & & 1. & & & & 0.072 \\
\hline 835 & 3 & 2 & 0.65 & & 0.05 & 4.91 & & & 2.29 & & & & 0. & & 0.094 \\
\hline DP10836 & 3 & 3 & 0.65 & & 0.0 & 3. & & & 1.5 & & & & & & .021 \\
\hline & 3 & 4 & 0.65 & & 0 & & & & 2.32 & & & & & & \\
\hline$D$ & 3 & 5 & 0.6 & & 0.02 & & 29 & $7 \cdot 37$ & 2.50 & & & & & & \\
\hline 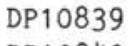 & 3 & 6 & 0.51 & & 0.03 & 5 & & & 2 & & 1 & & & & \\
\hline 0 & 4 & 1 & 0.36 & & 0. & & 29 & 9 & 2.35 & & & & & & 76 \\
\hline DP & 4 & 2 & 0.36 & & 0.02 & & & 7.72 & 2.28 & & & & & & 0.136 \\
\hline DP & 4 & 3 & 0.36 & & 0.03 & 6.36 & 31.3 & 7.73 & 2.42 & & 2.20 & & 0.046 & 0. & 0.341 \\
\hline DP10843 & 4 & 4 & 0.21 & 21 & 0.02 & 3. & & & 2.82 & & 1. & 0.2 & 0.040 & 0. & 0.104 \\
\hline DP10844 & 5 & 1 & 0.31 & & 0.0 & & & & 2. & & & & 0.0 & & 0.100 \\
\hline DP10 & 5 & 2 & 0.3 & & 0 & & & & 2. & & & & & & \\
\hline DP1 & 5 & 3 & 0. & & 0.06 & & & & 1 & & & & & & \\
\hline & 5 & 4 & 0.31 & & 0.03 & & & & 1 & & & & & & \\
\hline DP1 & 5 & 5 & 0.31 & & م & & & 8 & 2.14 & & & & 3 & 1 & 0.161 \\
\hline DP & 5 & 6 & 0. & & 0.02 & & & 7.92 & 2.39 & & & & 0. & & 1.335 \\
\hline DP10850 & 6 & 1 & 0.35 & & 0.02 & & & & 1.98 & 0.73 & & & 0.045 & & 0.067 \\
\hline & 6 & 2 & 0.35 & & 0.02 & 3. & 32.8 & 6.66 & 2.08 & 0. & 1.29 & & 0.042 & & 0.080 \\
\hline DP10852 & 6 & 3 & 0.35 & & 0.02 & & 29 & 7.26 & 2.18 & 0. & & & 0.044 & & 0.110 \\
\hline DP10853 & 6 & 4 & 0.35 & & 0.02 & & 31 & 7.88 & 2.36 & 0. & 1. & & 0.046 & 0. & 0.063 \\
\hline DP10854 & 6 & 5 & 0.3 & & 0.0 & 3. & & 6.72 & 2.07 & 0. & & 0. & 0.042 & & 0.074 \\
\hline DP10855 & 6 & 6 & 0.1 & & 0.0 & 2. & & & 1. & & & & 0.0 & & 0.046 \\
\hline DP108 & 7 & 1 & 0. & & 0 & & & & & & & & & & 0.248 \\
\hline DP1 & 7 & 2 & & & & & & 6.82 & 2.21 & 0.63 & 1.56 & 0. & 0. & & .255 \\
\hline DP10 & 7 & 3 & & & 0.02 & & & & 2.45 & 0.73 & 1.76 & & & & \\
\hline DP10859 & 7 & 4 & & & 0.01 & 3.35 & 32.1 & 7.04 & 2.35 & 0.48 & 1.45 & 0.312 & 0.042 & 0.091 & 0.223 \\
\hline DP10860 & 7 & 5 & & & 0.02 & 4.19 & 34.2 & 8.45 & 2.66 & 0.68 & 1.90 & 0.403 & 0.048 & 0.073 & 0.292 \\
\hline DP10861 & 7 & 6 & & & 0.02 & 3.98 & 31.9 & 6.85 & 1.85 & 1.10 & 1.43 & 0.328 & 0.041 & 0.072 & 0.338 \\
\hline DP10862 & 8 & 1 & 0.57 & & 0.01 & & & 6.57 & 1.94 & 0.74 & 1.28 & 0.314 & 0.046 & 0.078 & 0.077 \\
\hline DP10863 & 8 & 2 & 0.57 & & 0.0 & & & & 1.92 & 0.89 & 1.01 & & 0.043 & 0.084 & 0.106 \\
\hline DP10864 & 8 & 3 & 0.57 & & 0.01 & 1.96 & & 5.32 & 2.51 & 0.79 & 0.75 & 0.199 & 0.033 & 0.070 & 0.068 \\
\hline
\end{tabular}


Table 1. (Continued).

\begin{tabular}{|c|c|c|c|c|c|c|c|c|c|c|c|c|c|c|c|}
\hline $\begin{array}{c}\text { OSU } \\
\text { Lab } \\
\text { Sample }\end{array}$ & ذِ & $\begin{array}{l}\text { 듬 } \\
\stackrel{0}{0} \\
\mathscr{N}\end{array}$ & 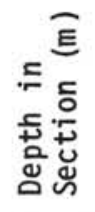 & 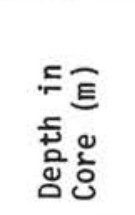 & $\mathrm{Mn}$ & $\mathrm{Fe}$ & $\mathrm{Si}$ & $\mathrm{A} 1$ & K & $\mathrm{Ca}$ & $\mathrm{Mg}$ & $\mathrm{Ti}$ & $P$ & $\mathrm{Ba}$ & $S$ \\
\hline J & 8 & 4 & 0.57 & & & 8 & & & & 2 & 9 & & 7 & & 00 \\
\hline P10866 & 8 & 5 & 0.57 & 87.07 & 0.01 & 2.53 & & & & 0.86 & & & .038 & .075 & 0.102 \\
\hline P10867 & 8 & 6 & 0.57 & 88.57 & 0.02 & 3.59 & 30.7 & 7.86 & 2.26 & 0.79 & 1. & 0.377 & 0.047 & 0.074 & 0.051 \\
\hline P10868 & 8 & 7 & 0.37 & 89.87 & 0.02 & 3.51 & 32.6 & 6.92 & 2.06 & 0.53 & 1.39 & 0.327 & 0.043 & 0.077 & 0.037 \\
\hline DP10869 & 9 & 1 & 1.23 & 91.23 & 0.04 & 3.19 & 32.5 & 7.21 & 2.21 & 0.63 & 1.27 & 0.304 & 0.041 & 0.077 & 0.022 \\
\hline P10870 & 9 & 2 & 0.91 & 92.41 & 0.04 & 3.34 & 32.3 & 6.52 & 1.89 & 0.65 & 1.16 & 0.288 & 0.039 & 0.076 & 0.019 \\
\hline P10871 & 9 & 3 & 0.91 & 93.91 & 0.01 & & 32.5 & 6.86 & 2.22 & 0.55 & 1.16 & 0.275 & & & 0.031 \\
\hline P10872 & 9 & 4 & 1.19 & & 0.05 & & & 7.02 & 2. & & 1. & & & & \\
\hline P10873 & 9 & 5 & 0.91 & & 0 & & & & & & 1. & & & & \\
\hline P10874 & 10 & 1 & 0.55 & 10 & 7 & & & & & & & & & & \\
\hline P10875 & 10 & 2 & 0.55 & 101.55 & 0. & & 30.4 & & & & & & & & 0.013 \\
\hline 76 & 10 & 3 & 0.55 & 103.05 & 0.05 & & 32.7 & 6.43 & 1.97 & & 1. & 0. & & & 0.023 \\
\hline DP 10877 & 10 & 4 & 0.55 & 104.55 & 0.04 & & 32.6 & 7 & 2.07 & & 1 & 24 & 39 & & 0.033 \\
\hline P10878 & 10 & 5 & 0.55 & 106.05 & 0.03 & & 32.0 & 6 & 1.99 & & 1.23 & 0.291 & 0.037 & 71 & 0.051 \\
\hline DP10879 & 11 & 1 & 0.99 & 109.99 & 0.08 & & 32.2 & 7.68 & 2.16 & 0.46 & 1.53 & 0.324 & 0.042 & 0.072 & 0.023 \\
\hline 880 & 11 & 2 & 0.99 & 111.49 & 0.05 & 3. & 33.1 & 6. & 1.94 & 0.47 & 1.35 & 0.299 & 0.039 & 85 & 0.027 \\
\hline DP10881 & 11 & 3 & 0.99 & 112.99 & 0.08 & & & 5. & 1.76 & 0.29 & 1. & 0.256 & 0.036 & 65 & 0.035 \\
\hline DP10882 & 11 & 4 & 0.99 & 114.49 & 0 & & & & & & & & & & \\
\hline P10883 & 11 & 5 & 0.71 & & & & & & & & & & & & \\
\hline 384 & 12 & 1 & 0.87 & & & & & & & & & & & & \\
\hline 85 & 12 & 2 & 0.87 & & & & 34.3 & & 2.11 & & & & & & \\
\hline 86 & 12 & 3 & 0.87 & 122.37 & 0.02 & 3.26 & 33.0 & 6. & 1.89 & 0.30 & & 0.279 & 0.036 & 93 & 0.059 \\
\hline 87 & 12 & 4 & 0.87 & 123.87 & 0.02 & 3. & 33.1 & 6.64 & 1.94 & 0.45 & 1 & 0.295 & 0.038 & 70 & 0.013 \\
\hline 88 & 12 & 5 & 0.85 & 125.35 & 0.01 & 3.27 & 32.2 & & 1.88 & 0.48 & 1.28 & 0.294 & 0.036 & 0.086 & 0.039 \\
\hline DP10889 & 12 & 6 & 0.07 & 126.07 & 0.04 & & 31.5 & & 1.83 & 0.95 & 1.30 & 0.306 & 0.041 & & 0.051 \\
\hline DP10890 & 13 & 1 & 0.42 & 128.42 & 0.02 & & 32.4 & & & 0.29 & & 0.192 & 0.034 & & 0.120 \\
\hline DP10891 & 13 & 2 & 0.42 & 129.92 & 0.07 & & & & & 0.27 & & & 0.0 & & 0.012 \\
\hline DP10892 & 13 & 3 & 0.42 & 131.42 & 0. & & & & & 0.29 & 1. & 0.2 & & 70 & 0.087 \\
\hline DP10893 & 13 & 4 & 0.42 & & 0. & & & & 1. & 0.31 & 1. & 0. & & & 0.055 \\
\hline & 13 & 5 & 0.42 & & & & & & 1. & & & & & & 0.060 \\
\hline DP10895 & 14 & 1 & 0.81 & 138.31 & 0.01 & 2.63 & 31.9 & & 1.83 & 0.25 & 0.90 & 0.238 & 0.033 & & 0.051 \\
\hline DP10896 & 14 & 2 & 0.81 & 139.81 & 0.05 & 3.02 & 33.6 & & 1.80 & 0.40 & 1.08 & 0.263 & 0.035 & 0.072 & 0.189 \\
\hline DP1 0897 & 14 & 3 & 0.81 & 141.31 & 0.02 & 3.01 & 33.8 & & 1.84 & 0.28 & 1.12 & 0.265 & 0.035 & 0.075 & 0.192 \\
\hline DP10898 & 14 & 4 & 0.81 & & 0.01 & & & & 1.45 & 0.25 & 0.70 & 0.162 & 0.026 & 0.053 & 0.190 \\
\hline DP10899 & 14 & 5 & 0.81 & 144.31 & 0.23 & 3.21 & & 7.07 & 2.09 & 0.36 & 1.30 & & 0.041 & 0.082 & 0.030 \\
\hline DP10900 & 15 & 1 & 0.89 & 147 & 0. & 4. & & & & & & & & & 0.020 \\
\hline DP10901 & 15 & 2 & 0.21 & 148.71 & 0.03 & & & 7.55 & 2.26 & 0.41 & & & 0.038 & 0.080 & 0.022 \\
\hline
\end{tabular}

Note: Samples DP10808-10819 are from Hole 579, subsequent samples are from Hole 579A.

580 contain higher and more variable amounts of $\mathrm{Mn}$ (Fig. 3). Given the gray green color of the Site 580 sediments, which implies $\mathrm{Fe}^{3+}$ reduction (Lyle, 1983), we are more inclined to assign the higher Mn values to the presence of $\mathrm{MnCO}_{3}$ than to oxyhydroxides. We have not been able to identify $\mathrm{MnCO}_{3}$ in X-ray diffractograms, because of its low concentration, but Coleman et al.'s (1982) description of $\mathrm{MnCO}_{3}$ in similar reduced sediments collected on Leg 68 (Pedersen and Price, 1982) and the availability of $\mathrm{CO}_{3}{ }^{2-}$ at Site 580 (inferred from the $\mathrm{Ca}$ concentrations) add credibility to this hypothesis.

At Site 581, the Mn profile can be divided into three segments (Fig. 4). Above $224 \mathrm{~m}$, in the gray green sediments, Mn values (with one exception) are very low, comparable to those at Sites 579 and 580 . In this interval, oxyhydroxide Mn has been reduced and lost from the sediments. The residual detrital $\mathrm{Mn}$ is somewhat diluted by biogenic opal. From 224 to $245 \mathrm{~m}$, in the light brown siliceous clay, some reduction may have taken place, but there is less opal dilution. From 245 to $276 \mathrm{~m}$, authigenic Mn oxyhydroxides form a much larger fraction of the dark brown pelagic clay, which accumulated more slowly and is strongly oxidizing. The Mn contents of the Site 581 cherts $(276-343 \mathrm{~m})$ are variable, but generally fairly high (comparable to the light brown siliceous clays), suggesting that the silicified clays were more like the sediments below $224 \mathrm{~m}$ than the shallower deposits at Site 581.

The elements $\mathrm{Ba}, \mathrm{P}$, and $\mathrm{Ca}$, which, like $\mathrm{Si}$, are associated with biogenic deep-sea deposition, have distributions intermediate between those of $\mathrm{Ti}-\mathrm{Al}$ and $\mathrm{Si}$. At Sites 579 and 580, P resembles $\mathrm{Ti}$, whereas $\mathrm{Ba}$ resembles $\mathrm{Si}$ (although values at Site 580 are distinctly higher and more variable than those at Site 579; Fig. 3). Ca has its own distribution, with high variability at both sites, and 
Table 2. Elemental composition (wt.\%) of Site 580 sediment samples.

\begin{tabular}{|c|c|c|c|c|c|c|c|c|c|c|c|c|c|c|c|}
\hline $\begin{array}{c}\text { OSU } \\
\text { Lab } \\
\text { Sample }\end{array}$ & ঠั่ & 등 & 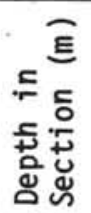 & 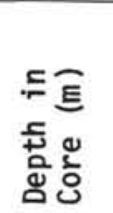 & $M n$ & $\mathrm{Fe}$ & $\mathrm{Si}$ & $\mathrm{Al}$ & $K$ & $\mathrm{Ca}$ & $\mathrm{Mg}$ & $\mathrm{Ti}$ & $P$ & $\mathrm{Ba}$ & $S$ \\
\hline DP10902 & 1 & 1 & 0.19 & 0.19 & 45 & 3.73 & 3 & 7.27 & 1.51 & 1.12 & 1.37 & 11 & 0.066 & 0.160 & 0.082 \\
\hline DP10903 & 1 & 1 & 0.91 & 0.91 & & & & 7.04 & & & & & & & 0.086 \\
\hline DP10904 & 1 & 2 & 0.21 & 1.71 & 0.17 & 4.15 & 30.8 & 7.15 & 1.75 & 1.29 & 1.79 & & 0.052 & & 0.099 \\
\hline DP10905 & 2 & 1 & 0.56 & 3.86 & 0.18 & 4.42 & 30.8 & 7.56 & 2.16 & 0.82 & 1.95 & 0.355 & 0.054 & 0.119 & 0.072 \\
\hline DP10906 & 2 & 2 & 0.56 & 5.36 & 0.14 & 4.85 & 31.6 & 6.81 & 1.91 & 0.74 & 1.86 & & 0.049 & 0.105 & 0.251 \\
\hline DP10907 & 2 & 3 & 0.56 & 6.86 & 0.08 & 4.10 & 31.8 & 7.71 & 2.06 & 0.78 & 1.70 & 0.353 & 0.049 & 0.138 & 0.169 \\
\hline DP10908 & 2 & 4 & 0.56 & 8.36 & 0.18 & 5.67 & 28.0 & 6.51 & 1.82 & 1.15 & 2.10 & & 0.047 & 0.081 & 0.101 \\
\hline DP10909 & 2 & 5 & 0.56 & 9.86 & 0.16 & 4.02 & 31.0 & 7.46 & 2.05 & & 1.79 & & 0.053 & & 0.110 \\
\hline DP10910 & 2 & 6 & 0.56 & 11.36 & 0.16 & 4.65 & & & & & & & & & 0.137 \\
\hline DP10911 & 3 & 1 & 0.81 & 13.61 & 0.10 & 4.19 & 30.2 & 8.31 & 2.28 & 0.88 & 1.91 & & & & 0.126 \\
\hline DP10912 & 3 & 2 & 0.81 & 15.11 & 0.12 & 4.16 & 30.5 & 7.80 & 2.20 & 0.77 & 1.90 & 0. & 0.050 & 0.1 & 0.072 \\
\hline DP10913 & 3 & 3 & 0.91 & 16.71 & 0.13 & 4.21 & 30.0 & & 2.39 & 1.22 & 1.99 & & 0. & 5 & 0.220 \\
\hline DP10914 & 3 & 4 & 0.76 & 18.06 & 0.20 & 3.93 & 31.1 & & 2.34 & 0.75 & 1.80 & & 0. & & 0.068 \\
\hline DP10915 & 3 & 5 & 0.91 & 19.71 & 0.05 & 3.56 & 31.1 & & 2.61 & 0.90 & 1.63 & & 0. & & 0.043 \\
\hline DP10916 & 3 & 6 & 0.76 & 21.06 & 0.10 & 5.03 & 30.6 & & 2.40 & 0.69 & 2.10 & & 52 & 0. & 0.330 \\
\hline DP10917 & 4 & 1 & 0.45 & 22.75 & 0.07 & 4.03 & 32.4 . & 7.33 & 1.80 & 1. & 1.40 & & & & 0.066 \\
\hline DP10918 & 4 & 2 & 0.45 & 24.25 & 0 . & 4. & 30.6 & & 2. & 8 & 1.99 & & 0. & & 0.269 \\
\hline DP10919 & 4 & 3 & 0.45 & 25.75 & 0.10 & 4.60 & 30.2 & 8.42 & 2.46 & 0.74 & 2.05 & 0. & & & 0.255 \\
\hline DP10920 & 4 & 4 & 0.45 & 27.25 & 0.09 & 4.01 & 32.1 & 7.32 & 2.01 & 0.72 & 1.59 & & 0. & & 0.076 \\
\hline DP 10921 & 4 & 5 & 0.45 & 28.75 & 8 & 4. & 30 & & 2.34 & 1 & 1.96 & & 0 & & 0.101 \\
\hline DP10922 & 4 & 6 & 0.45 & 30.25 & 0.06 & 3. & 31.2 & 3 & 2.10 & 0.87 & 1.73 & & 0 . & & 0.080 \\
\hline DP10991 & 5 & 1 & 1.06 & 32.86 & 0.17 & 3.28 & 30.0 & 6.82 & 1.86 & 1.15 & 1.51 & & 0. & & 0.152 \\
\hline DP10992 & 5 & 2 & 1.06 & $34 \cdot 36$ & 0.14 & 3. & 29.5 & 7.00 & 2.38 & 0.95 & 1.43 & & & & 0.141 \\
\hline DP10993 & 5 & 3 & 1.06 & 35.86 & 0.07 & 4. & 30.4 & 8. & 2.42 & 2 & 94 & 0. & 50 & & 0.069 \\
\hline DP10994 & 5 & 4 & 1.06 & 37.36 & 0.20 & 4.28 & 29.2 & 7.98 & 2.28 & 1. & 1. & 0. & 0. & & 0.279 \\
\hline DP10995 & 5 & 5 & 1.06 & 38.86 & 0.08 & 3.61 & 30.5 & 7.74 & 2.19 & 0. & 1.61 & & 0.0 & & 0.214 \\
\hline DP1C & 6 & 1 & 0.31 & 41.61 & 0.14 & & 30.6 & 7.84 & 2.17 & 1. & 1.82 & 58 & 0.0 & & 0.373 \\
\hline DP10924 & 6 & 2 & 0.31 & 43.11 & 5 & 3. & 32.7 & 7.52 & 2 & 9 & 1.61 & 0. & 0. & & 58 \\
\hline DP10 & 6 & 3 & 0.31 & 44.61 & 0 & 3. & 29.7 & 7.51 & 2. & 0.92 & 38 & & 0. & & 0.215 \\
\hline DP10926 & 6 & 4 & 0.31 & 46.11 & 0.09 & 3. & 29.8 & 7. & 2.40 & 0.70 & 35 & & 47 & & 0.105 \\
\hline DP10927 & 6 & 5 & 0.31 & 47.61 & 0.17 & 5.46 & 30.4 & & 2.09 & 1.01 & 1.77 & & 49 & 2 & 0.197 \\
\hline DP10928 & 6 & 6 & 0.31 & 49.11 & 0.06 & 4.28 & 31.2 & 8.26 & 2.45 & 0.79 & 1.86 & & 0. & & 0.088 \\
\hline DP10929 & 7 & 1 & 0.20 & 51.00 & 0.13 & 4. & 31.9 & 7.83 & 2.21 & 0.66 & 1.90 & 0. & 0.048 & 0. & 0.170 \\
\hline DP10930 & 7 & 2 & 0.20 & 52.50 & 0.11 & & & & 2.29 & 0.60 & 1.99 & & 0.049 & 0. & 0.359 \\
\hline DP10931 & 7 & 3 & 0.20 & 54.00 & 0.11 & & 30.8 & 8.55 & 2.38 & 0. & 1.99 & & 0. & & 0.050 \\
\hline DP10 & 7 & 4 & 0.20 & 55.50 & 3 & 3 & 31.0 & 8.10 & 2.24 & 0.91 & 1.90 & & 0. & & 0.241 \\
\hline DP & 7 & 5 & 0.20 & 5 & 0 & 4 & 3 & 2 & 1 & 0 & 1.48 & & 0.046 & & 0 \\
\hline 0934 & 8 & 1 & 0.42 & 60.72 & 0 & 5. & 37.0 & 9.03 & 2.66 & 1.31 & 4 & & 0.067 & & 0.073 \\
\hline DP10935 & 8 & 2 & 0.42 & 62.22 & 0.16 & 3.20 & 31.9 & 6.63 & 1.77 & 0.85 & 1.38 & 0 . & 0.042 & & 0.169 \\
\hline DP10936 & 8 & 3 & 0.42 & 63.72 & 0.09 & 3.50 & 33.3 & 6.76 & 1.88 & 0.84 & 1.45 & 0.304 & 0.045 & 0. & 0.129 \\
\hline DP10937 & 8 & 4 & 0.42 & 65.22 & 0.01 & 2.81 & 32. & 6.64 & 2.51 & 0.48 & 1.13 & 0.2 & 0.038 & 0.124 & 0.035 \\
\hline DP10938 & 8 & 5 & 0.42 & 66.72 & 0.06 & & 32 & 6. & 1. & 1.06 & 1.32 & & 0.045 & 0. & 0.085 \\
\hline DP10939 & 8 & 6 & 0.42 & 68.22 & & & & & 2. & 0 . & 3 & & 0.0 & 0 . & 0.128 \\
\hline DP10940 & 9 & 1 & 0.54 & & 0 & & 30.9 & 7.10 & 2. & 0.62 & 1.97 & & 0. & 0. & 0.470 \\
\hline DP 10 & 9 & 2 & 0.54 & & 6 & 2 & 30.8 & 6.48 & 2.22 & 0.84 & 1.14 & & 40 & & 0.16 \\
\hline DP109 & 9 & 3 & 0.54 & & 0 & & 30.9 & 7.55 & 2.29 & 0.69 & 1.79 & 0 . & 0. & 0. & 0.193 \\
\hline DP10943 & 9 & 4 & 0.54 & & 0.16 & 4. & 32.9 & 6.76 & 1.97 & 0.86 & 1.54 & 0.294 & 0.043 & & 0.104 \\
\hline DP10944 & 9 & 5 & 0.54 & 76.34 & 0.19 & 3. & 32.4 & 6.97 & 2.00 & 0.62 & 1.50 & 0.321 & 0.045 & 0.120 & 0.059 \\
\hline DP10945 & 10 & 1 & 0.41 & 79.71 & 0.0 & 3. & 31.1 & 7.28 & 2.18 & 0.59 & 1.70 & & 0.046 & 0.101 & 0.267 \\
\hline DP10946 & 10 & 2 & 0.41 & & 0.1 & & 34. & 5.92 & 1.81 & 0.48 & 1.49 & 0.283 & 0.042 & 0.092 & 0.898 \\
\hline DP 10947 & 10 & 3 & 0.41 & & 0.2 & & & & 2.25 & & 2.16 & & 0.045 & 0.095 & 0.241 \\
\hline DP10948 & 10 & 4 & 0.41 & & & & 32.9 & 5.82 & 1.71 & & 1.31 & & 0.040 & 0.112 & 0.02 \\
\hline DP10949 & 10 & 5 & 0.41 & 85.71 & 0.40 & 6.14 & 32.6 & & 1.84 & 0.49 & 2.12 & 0.2 & 0.040 & & 0.26 \\
\hline DP10950 & 10 & 6 & 0.41 & 87.21 & 0.28 & 4.17 & 33.2 & 7.82 & 2.31 & 0.72 & 1.86 & 0.362 & 0.050 & 0.116 & 0.13 \\
\hline DP10951 & 11 & 1 & 0.44 & 89.24 & 0.06 & 2.94 & 32.9 & 5.63 & 1.54 & 0.89 & 1.11 & 0.265 & 0.041 & 0.101 & 0.26 \\
\hline DP10952 & 11 & 2 & 0.44 & 90.74 & 0.07 & 3.36 & 33 & 6.37 & 1.84 & 0. & 1.38 & 0.287 & 0.041 & 0.124 & 0.275 \\
\hline DP10953 & 11 & 3 & 0.44 & 92.24 & 0.06 & & 32.8 & 6.81 & 1.97 & 0 . & 1.58 & 0.288 & 0.041 & 0.093 & 3.04 \\
\hline
\end{tabular}


Table 2. (Continued).

\begin{tabular}{|c|c|c|c|c|c|c|c|c|c|c|c|c|c|c|c|}
\hline $\begin{array}{c}\text { OSU } \\
\text { Lab } \\
\text { Sample }\end{array}$ & ذั่ & 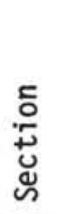 & 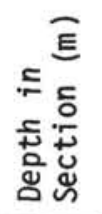 & 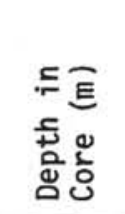 & $\mathrm{Mn}$ & $\mathrm{Fe}$ & Si & A1 & K & $\mathrm{Ca}$ & $\mathrm{Mg}$ & $\mathrm{Ti}$ & $\mathbf{P}$ & $\mathrm{Ba}$ & $S$ \\
\hline DP10954 & 11 & 4 & 0.44 & 93.74 & 0.07 & 3.60 & 32.4 & 6.66 & 2.13 & 0.61 & 1.58 & 0.322 & 0.043 & 0.088 & 0.351 \\
\hline DP10955 & 11 & 5 & 0.44 & 95.24 & 0.22 & 3.19 & 34.8 & 6.15 & 1.89 & 0.54 & 1.43 & 0.290 & 0.041 & 0.131 & 0.124 \\
\hline DP10956 & 12 & 1 & 1.11 & 99.41 & 0.07 & 2.56 & 33.9 & 5.01 & 1.71 & 0.43 & 1.03 & 0.227 & 0.036 & 0.087 & 0.231 \\
\hline DP10957 & 12 & 2 & 1.11 & 100.91 & 0.13 & 2.82 & 33.5 & 4.34 & 1.43 & 1.49 & 1.14 & 0.219 & 0.035 & 0.093 & 0.486 \\
\hline DP10958 & 12 & 3 & 0.81 & 102.11 & 0.09 & 2.58 & 34.2 & 5.23 & 2.01 & 0.49 & 1.10 & 0.226 & 0.037 & 0.111 & 0.144 \\
\hline DP10959 & 12 & 4 & 1.11 & 103.91 & 0.03 & 2.83 & 33.8 & 5.29 & 1.64 & 0.37 & 1.12 & 0.242 & 0.038 & 0.127 & 0.132 \\
\hline DP10960 & 12 & 5 & 0.96 & 105.26 & 0.14 & 4.05 & 32.3 & 7.43 & 2.29 & 0.67 & 1.85 & 0.352 & 0.045 & 0.071 & 0.525 \\
\hline DP10961 & 12 & 6 & 1.11 & 106.91 & 0.14 & 3.19 & 33.8 & 6.12 & 1.92 & 0.78 & 1.32 & 0.279 & 0.042 & 0.108 & 0.145 \\
\hline DP10962 & 13 & 1 & 1.11 & 108.91 & 0.06 & 3.42 & 33.6 & 4.78 & 1.56 & 0.33 & 1.14 & 0.224 & 0.035 & 0.116 & 0.516 \\
\hline DP10963 & 13 & 2 & 1.11 & 110.41 & 0.09 & 2.89 & 36.4 & 4.25 & 1.43 & 0.26 & 1.04 & 0.202 & 0.038 & 0.119 & 0.103 \\
\hline DP10964 & 13 & 3 & 1.11 & 111.91 & 0.08 & 2.72 & 35.3 & 4.37 & 1.46 & 0.28 & 1.07 & 0.214 & 0.038 & 0.121 & 0.089 \\
\hline DP10965 & 13 & 4 & 1.11 & 113.41 & 0.09 & 3.38 & 34.0 & 5.66 & 1.49 & 1.22 & 1.13 & 0.256 & 0.042 & 0.062 & 0.315 \\
\hline DP10966 & 13 & 5 & 1.11 & 114.91 & 0.12 & 3.02 & 34.0 & 5.42 & 1.71 & 0.45 & 1.27 & 0.238 & 0.038 & 0.126 & 0.255 \\
\hline DP10967 & 13 & 6 & 1.11 & 116.41 & 0.12 & 3.44 & 31.6 & 6.02 & 1.89 & 1.39 & 1.44 & 0.280 & 0.040 & 0.082 & 0.532 \\
\hline DP10968 & 14 & 1 & 1.16 & 118.46 & 0.14 & 3.83 & & 5.32 & 1.64 & 0.64 & 1.39 & 0.252 & 0.038 & 0.120 & 1.450 \\
\hline DP10969 & 14 & 2 & 1.16 & 119.96 & 0.11. & 2.38 & 33.6 & 4.84 & 1.65 & 0.90 & 0.86 & 0.2 & 0.0 & 0.085 & 0.117 \\
\hline DP10970 & 14 & 3 & 1.16 & 121.46 & 0.05 & 3.65 & 32.9 & 6.31 & 1.96 & 0.46 & 1.49 & 0.280 & 0.040 & 0.130 & 0.202 \\
\hline DP10971 & 14 & 4 & 1.16 & 122.96 & 0.15 & 3.21 & 35.2 & 4.87 & 1.55 & 0.29 & 1.24 & 0.233 & 0.036 & 0.130 & 0.231 \\
\hline DP10972 & 14 & 5 & 1.16 & 124.46 & 0.09 & 3.12 & 34.0 & 5.73 & 1.80 & 0.42 & 1.29 & 0.278 & 0.039 & 0.103 & 0.243 \\
\hline DP10973 & 14 & 6 & 1.16 & 125.96 & 0.12 & 3.10 & 33.4 & 5.66 & 1.85 & 0.32 & 1.43 & 0.267 & 0.037 & 0.116 & 0.099 \\
\hline DP10974 & 15 & 1 & 0.58 & 127.38 & 0.10 & 3.53 & 32.3 & 6.65 & 2.12 & 0.41 & 1.57 & 0.302 & 0.039 & 0.103 & 0.151 \\
\hline DP10975 & 15 & 2 & 0.58 & 128.88 & 0.05 & 2.54 & 33.7 & 5.16 & 1.71 & 0.53 & 0.91 & 0.212 & 0.035 & 0.105 & 0.002 \\
\hline DP10976 & 15 & 3 & 0.58 & 130.38 & 0.08 & 2.70 & & 5.13 & 1.60 & 0.40 & 1.07 & 0.225 & 0.034 & 0.089 & 0.044 \\
\hline DP10977 & 15 & 4 & 0.58 & 131.88 & 0.10 & 3.09 & & 5.49 & 1.74 & 0.33 & 1.19 & 0.255 & 0.037 & 0.114 & 0.196 \\
\hline DP10978 & 15 & 5 & 0.58 & 133.38 & 0.08 & 2.82 & & & 1.77 & 0.60 & 1.05 & 0.253 & 0.037 & 0.083 & 0.012 \\
\hline DP10979 & 15 & 6 & 0.58 & 134.88 & 0. & 2. & & & 2. & 0. & 0. & 0.185 & 0.033 & 0.075 & 0.019 \\
\hline DP10980 & 16 & 1 & 0.81 & 137.11 & 0.14 & 3.70 & 33.5 & 5.76 & 1.75 & 0.24 & 1. & 0. & 0. & 0. & 0.005 \\
\hline DP10981 & 16 & 2 & 0.81 & 138.61 & 0.13 & 3.20 & 41.2 & 5.43 & 1.84 & 0.35 & 1.32 & 0.277 & 0.045 & 0.113 & 0.150 \\
\hline DP10982 & 16 & 3 & 0.81 & 140.11 & 0.12 & 2.53 & 35.4 & 4.72 & 1.53 & 0.30 & 1.11 & 0.221 & 0.034 & 0.103 & 0.033 \\
\hline DP10983 & 16 & 4 & 0.81 & 141.61 & 0.05 & 2.94 & 34.7 & 5.48 & 1.69 & 0.33 & 1.18 & 0.239 & 0.037 & 0.092 & 0.012 \\
\hline DP1 0984 & 16 & 5 & 0.81 & 143.11 & 0.05 & 2.45 & 35.3 & 3.06 & 1.06 & 0.08 & 0.72 & 0.152 & 0.030 & 0.092 & 0.212 \\
\hline DP10985 & 16 & 6 & 0.81 & 144.61 & 0.05 & 2.27 & 34.8 & 4.18 & 1.44 & 0.21 & 0.90 & 0.203 & 0.031 & 0.080 & 0.032 \\
\hline DP10986 & 17 & 1 & 0.78 & 146.58 & 0.06 & 2.31 & 34.5 & 4.37 & 1.59 & 0.39 & 0.77 & 0.190 & 0.035 & 0.111 & 0.000 \\
\hline DP10987 & 17 & 2 & 0.78 & 148.08 & 0.05 & 2.88 & & 4.85 & 1.53 & 0.27 & 1.11 & 0.229 & 0.031 & 0.123 & 0.079 \\
\hline DP10988 & 17 & 3 & 0.78 & 149.58 & 0.03 & 2.59 & & 4.93 & 1.67 & 0.27 & 1.03 & 0.220 & 0.033 & 0.129 & 0.038 \\
\hline DP10989 & 17 & 4 & 0.78 & 151.08 & 0.04 & 2.79 & & 6.01 & 2.11 & 0.48 & 1.14 & 0.236 & 0.034 & 0.096 & 0.065 \\
\hline DP10990 & 17 & 5 & 0.78 & 152.58 & 0.11 & 2.96 & 33.3 & 5.76 & 1.73 & 0.53 & 1.22 & 0.262 & 0.035 & 0.096 & 0.103 \\
\hline
\end{tabular}

a well-defined decrease in concentration below about $3.2 \mathrm{~m} . y .(110 \mathrm{~m})$ at Site 579 and about $2.3 \mathrm{~m} . y .(100 \mathrm{~m})$ at Site 580 .

At Site $581, \mathrm{Ba}, \mathrm{P}$, and $\mathrm{Ca}$ all resemble Ti rather than $\mathrm{Si}$ in the clay section. In the basal dark brown pelagic clay $(245-276 \mathrm{~m})$ and in the upper and lower cherts (below $276 \mathrm{~m}$ ), however, $\mathrm{P}$ has clear peaks that are matched by $\mathrm{Ca}$ peaks, suggesting abundant fish debris. $\mathrm{Ba}$ also peaks in the lower cherts, but not the upper ones. Ba peaks in the oxidized (light and dark brown) pelagic clays, as it does elsewhere in the Pacific (Dymond, 1981; Leinen, 1979).

Because elements like silicon are derived from more than one source (detrital aluminosilicates and biogenic opal, for example), we have carried out a Q-mode factor analysis of the data. In its conventional form (Klovan and Imbrie, 1971), such a factor analysis expresses each sample in terms of a number of orthogonal functions that share the variance of the data set as equally as possible. This procedure simplifies the interpretation of large data sets, but the factors may be difficult to interpret physically because they can contain negative contributions of some elements.

Figure 5 shows the downhole variation in three factors derived from the data sets for both Sites 579 and 580. Overall, it is clear that the sediments at Site 579 are markedly less variable than those at Site 580 . The variability is due primarily to Mn (Factor 2), which is relatively uniform at Site 579 (particularly above $80 \mathrm{~m}$ ), but fluctuates markedly at Site 580 (Fig. 3). In contrast, the biogenically dominated Factor 3 (loaded in $\mathrm{Si}$ and $\mathrm{Ba}$; Fig. 5) is of comparable importance at the two sites. In fact, this factor is slightly more variable at Site 579 .

The factors are easier to interpret if the Q-mode axes are rotated toward the mean of the data set until they enter the positive quadrant (i.e., until their projections 
Table 3. Elemental composition (wt.\%) of Site 581 sediment samples.

\begin{tabular}{|c|c|c|c|c|c|c|c|c|c|c|c|c|c|c|c|}
\hline $\begin{array}{c}\text { OSU } \\
\text { Lab } \\
\text { Sample }\end{array}$ & ذั่ & 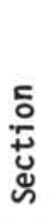 & 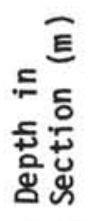 & 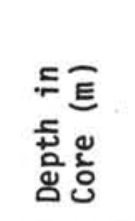 & $M n$ & $\mathrm{Fe}$ & Si & A1 & K & $\mathrm{Ca}$ & $\mathrm{Mg}$ & $\mathrm{Ti}$ & $P$ & $\mathrm{Ba}$ & $S$ \\
\hline DP10996 & 1 & 1 & 0.51 & 0.51 & 0.08 & 3.73 & 32.3 & 6.43 & 1.61 & 1.19 & 1.43 & 0.320 & 0.048 & 0.113 & 0.094 \\
\hline DP10997 & 2 & 1 & 0.37 & 181.87 & 0.09 & 2.71 & & & 1.51 & & 1.09 & & & & 0.137 \\
\hline DP10998 & 2 & 2 & 0.37 & 183.37 & 0.13 & 3.40 & 35.8 & 3.53 & 1.24 & 0.05 & 1.31 & 0.172 & 0.030 & 0.157 & .149 \\
\hline DP10999 & 2 & 3 & 1.28 & 185.78 & 0.08 & 3.34 & 40.3 & 5.72 & 1.90 & 0.15 & 1.43 & 0.274 & 0.039 & .157 & 0.085 \\
\hline DP1 1000 & 2 & 4 & 1.28 & 187.28 & 0.04 & 2.26 & 35.8 & 3.24 & 1.15 & 0.02 & 0.82 & 0.161 & 0.029 & .167 & 0.065 \\
\hline DP11001 & 2 & 5 & 0.37 & 187.87 & 0.11 & 2.65 & 35.3 & 4.17 & 1.43 & 0.22 & 1.01 & 0.214 & 0.033 & 0.162 & 0.050 \\
\hline DP11002 & 2 & 6 & 0.35 & 189.35 & 0.06 & 3.19 & 33.5 & 4.96 & 1.66 & 0.13 & 1.19 & 0.227 & 0.032 & 0.115 & 0.045 \\
\hline DP11003 & 2 & 7 & 0.16 & 190.66 & 0.01 & 2.84 & & & 1.71 & & 1.15 & & 0.032 & & 0.059 \\
\hline DP11004 & 3 & 1 & 0.22 & 191.22 & 0.05 & 2.34 & 34.1 & 4.53 & 1.62 & & 1.12 & & & & .109 \\
\hline DP11005 & 3 & 2 & 0.22 & 192.72 & 0.08 & 2.76 & 32.4 & 5.55 & 1.88 & & 8 & 44 & 0.034 & & .142 \\
\hline DP11006 & 3 & 3 & 0.22 & 194.22 & 0.13 & 2.07 & 34.9 & 3. & 1.31 & 0.03 & 0.92 & 87 & 0.032 & 5 & .096 \\
\hline DP11007 & 3 & 4 & 0.22 & 195.72 & 0.05 & 2.84 & 34.4 & & 1.69 & 0. & 1.17 & & & & 44 \\
\hline DP11008 & 3 & 5 & 0.22 & 197.22 & 0.07 & 4.34 & 35.2 & & 1.26 & 0.01 & 1.27 & & 30 & 0. & 0.040 \\
\hline DP11009 & 3 & 6 & 0.22 & 198.72 & 0.00 & 2.26 & & & 1.35 & 0.02 & 0.83 & 86 & 31 & & 0.030 \\
\hline DP11010 & 4 & 1 & 0.17 & 200.67 & 0.01 & 1.50 & & & 1.05 & 0. & 0.57 & & & & .081 \\
\hline DP11011 & 4 & 2 & 0.17 & 202.17 & 0.08 & 2.62 & 35 & 4.10 & 1.40 & 0.06 & 1.00 & & & & .033 \\
\hline DP11012 & 4 & 3 & 0.17 & 203.67 & 0.07 & 2.57 & 34 & & 1.30 & 0.0 & 0.90 & 87 & 2 & & 52 \\
\hline DP11013 & 4 & 4 & 0.17 & 205.17 & 7 & 3. & 34 & 7 & 34 & 0. & 1.45 & & & & 50 \\
\hline DP11014 & 4 & 5 & 0.17 & 206.67 & 0.04 & 3. & 35.6 & & 1.75 & 0. & 19 & & & & 58 \\
\hline DP1 1015 & 4 & 6 & 0.17 & 208.17 & 0.06 & 3.56 & 33.1 & & 1.86 & 0. & 1.33 & & & & 36 \\
\hline DP11016 & 4 & 7 & 0.17 & 209.67 & 0.04 & 3.96 & & & 1.60 & 0.05 & 1.30 & 0. & 32 & & .050 \\
\hline DP11017 & 5 & 1 & 0.41 & 210.41 & 0.04 & 3.06 & 36 & 4.82 & 1.58 & 0.07 & 1.04 & 0. & & & .028 \\
\hline DP1 1018 & 5 & 2 & 0.41 & 211.91 & 1.31 & 2.19 & & 3.72 & 1.28 & 0. & 0.82 & & & & .029 \\
\hline DP11019 & 5 & 3 & 0.41 & 213.41 & & 1.94 & & & 1.39 & & 0.85 & & & & 055 \\
\hline DP11020 & 5 & 4 & 0.41 & 214.91 & & 2.09 & & & 1.41 & & 0.88 & & & & \\
\hline DP11021 & 6 & 1 & 0.91 & 220.41 & 6 & $3 \cdot 33$ & 32 & 5.03 & 1.66 & 0 & 1.04 & 4 & 37 & & \\
\hline DP11022 & 6 & 2 & 0.45 & 221.45 & 7 & 3. & 34 & 5.12 & 1.73 & 0. & 1 & & & & 29 \\
\hline DP1 1023 & 6 & 2 & 1.18 & 222.18 & 0.07 & 3.10 & 33.8 & 4.50 & 1.53 & 0.15 & 0.93 & & & & 0.039 \\
\hline DP11024 & 6 & 3 & 0.45 & 222.95 & 0.07 & 4.08 & 30.8 & 6.51 & 2.08 & 0.34 & 1.31 & 0. & 7 & & 0.030 \\
\hline DP11025 & 6 & 3 & 1.18 & 223.68 & 0.11 & 4.11 & & & 2.09 & & 1.31 & & & & 0.018 \\
\hline DP11026 & 6 & 4 & 0.45 & 224.45 & & 4. & & & 2. & & 1. & & & & 21 \\
\hline DP11 & 6 & 4 & 1.18 & 225.18 & & 3. & & & & & & & & & \\
\hline DP1 1028 & 6 & 5 & 0.45 & 225.95 & 0. & 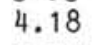 & & 1 & 2 & 4 & 30 & 3 & 6 & & 23 \\
\hline DP11029 & 6 & 5 & 1.18 & 226.68 & 0 . & 3.77 & & 6 & 2.01 & 0. & 1.20 & & 6 & & 0.021 \\
\hline DP1 1030 & 6 & 6 & 0.45 & 227.45 & 0.16 & 3.69 & 32 & 6.35 & 2.00 & 0. & 1.18 & 0.269 & & 0. & 0.052 \\
\hline DP1 1031 & 6 & 6 & 1.06 & 228.06 & 0.29 & 3.42 & 32.1 & 5.95 & 1.90 & 0.26 & 1.11 & 0.253 & 0.040 & & 0.044 \\
\hline DP11032 & 7 & 1 & 0.49 & 229.49 & 0.36 & 3.44 & 34.6 & 5.89 & 1.86 & 0. & 1.09 & 0.259 & 0.042 & & 0.025 \\
\hline DP1 1033 & 7 & 1 & 1.11 & 230.11 & 0.40 & 3. & & & 1. & & 1. & 0. & & & 59 \\
\hline DP11034 & 7 & 2 & 0.49 & 230.99 & 0.22 & & & 7.04 & & & 1.27 & 93 & & & 039 \\
\hline DP11035 & 7 & 2 & 1.11 & 231.61 & 0.21 & 3.75 & 31.7 & 6.31 & 2.00 & 0 & 1.14 & 7 & 39 & & 29 \\
\hline DP11036 & 7 & 3 & 0.49 & 232.49 & 0. & & & & 2.15 & 0.32 & 1.29 & DO & 0.039 & 0. & 0.046 \\
\hline DP11037 & 7 & 3 & 1.11 & 233.11 & 0.20 & & 30.5 & 6.54 & 2.08 & 0.21 & 1.16 & 0.288 & 0.037 & 0.143 & 0.024 \\
\hline DP11038 & 7 & 4 & 0.49 & 233.99 & 0.35 & 3.81 & 30.5 & 6.63 & 2.09 & 0.21 & 1.19 & 0.283 & 0.035 & 0.152 & 0.030 \\
\hline DP11039 & 7 & 4 & 1.11 & 234.61 & 0.23 & 3.8 & 31.0 & 6.50 & 2.12 & 0. & 1.15 & 0.283 & 0.038 & 0. & 0.025 \\
\hline DP11040 & 7 & 5 & 0.49 & 235.49 & 0.42 & 3. & & 5. & 1. & 0 . & 1. & 0 . & & & 0.073 \\
\hline DP11041 & 7 & 5 & 1.11 & 236.11 & 0.28 & & & & 2.06 & 0. & 1. & & & & 0.053 \\
\hline DP11042 & 7 & 6 & 0.49 & 236 & 0.32 & & 30.6 & 6.32 & 1.98 & 0.23 & & & & & 0.032 \\
\hline DP11043 & 7 & 6 & 1. & 237.61 & & & 30.7 & 6.54 & 2.30 & 0.31 & 1.08 & 0.253 & 0.037 & 0.109 & 0.019 \\
\hline DP11044 & 8 & 1 & 0.29 & 238.79 & 0.28 & 4.05 & 30.0 & 7.01 & 2.20 & 0.34 & 1.28 & 0.301 & 0.045 & 0.211 & 0.054 \\
\hline DP11045 & 8 & 1 & 1.01 & 239.51 & 0.21 & 4.01 & 30.4 & 6.95 & 2.16 & 0.32 & 1.28 & 0.292 & 0.049 & 0.203 & 0.053 \\
\hline DP11046 & 8 & 2 & 0.29 & 240.29 & 0.30 & 4.13 & 30.1 & 7.04 & 2.21 & & 1.28 & 0.302 & 0.046 & 0.218 & \\
\hline DP11047 & 8 & 2 & 1.01 & 241.01 & 0.32 & 3.79 & & & 2.10 & & 1.20 & 0.286 & 0.044 & 0.182 & 0.042 \\
\hline DP11 1048 & 8 & 3 & 0.29 & 241.79 & 0.27 & 3. & & 5.72 & 1.86 & & 1.03 & 0.248 & 0.036 & 0.183 & 0.055 \\
\hline DP11049 & 8 & 3 & 1.01 & 242.51 & 0.40 & 3. & & 6.97 & 2.15 & 0.23 & 1.18 & 0.282 & 0.039 & & 0.032 \\
\hline DP11050 & 8 & 4 & 0.29 & 243.29 & 0.41 & & & 6.37 & 2.03 & 0.17 & 1.16 & 0.271 & 0.041 & 0.236 & 0.057 \\
\hline DP11051 & 8 & 4 & 1.01 & 244.01 & & & 29.9 & 8.12 & 2.33 & 0.31 & 1.65 & 0.335 & 0.046 & 0.160 & 0.047 \\
\hline DP11052 & 8 & 5 & 0.29 & 244.79 & 0.64 & 4.74 & 27.0 & 8.18 & 2.31 & 0.31 & 1.86 & 0.350 & 0.050 & 0.109 & 0.021 \\
\hline
\end{tabular}


Table 3. (Continued).

\begin{tabular}{|c|c|c|c|c|c|c|c|c|c|c|c|c|c|c|c|}
\hline $\begin{array}{c}\text { OSU } \\
\text { Lab } \\
\text { Sample }\end{array}$ & ذั่ & 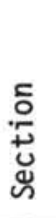 & 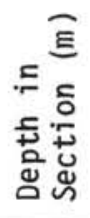 & 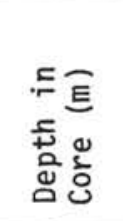 & $M n$ & $\mathrm{Fe}$ & Si & Al & K & $\mathrm{Ca}$ & $\mathrm{Mg}$ & $\mathrm{Ti}$ & $P$ & $\mathrm{Ba}$ & $S$ \\
\hline DP11053 & 8 & 5 & 1.01 & 245.51 & 0.61 & 4.86 & 23.8 & 7.19 & 2.06 & 0.30 & 1.71 & 0.342 & 0.043 & 0.174 & 0.036 \\
\hline DP11054 & 9 & 1 & 0.25 & 248.25 & 0.60 & 4.97 & 25.5 & 7.81 & 2.21 & 0.32 & 1.93 & 0.357 & 0.051 & 0.198 & 0.045 \\
\hline DP11055 & 9 & 1 & 0.73 & 248.73 & 0.90 & 4.66 & 24.9 & 7.62 & 2.02 & 0.27 & 1.90 & 0.327 & 0.043 & 0.162 & 0.033 \\
\hline DP11056 & 9 & 1 & 1.29 & 249.29 & 0.75 & 4.73 & 23.8 & 7.37 & 2.07 & 0.29 & 1.81 & 0.332 & 0.051 & 0.121 & 0.016 \\
\hline DP1 1057 & 9 & 2 & 0.25 & 249.75 & 0.87 & 4.97 & 23.8 & 7.29 & 2.09 & 0.33 & 1.79 & 0.343 & 0.047 & 0.149 & 0.030 \\
\hline DP11058 & 9 & 2 & 0.73 & 250.23 & 0.93 & 4.84 & 27.6 & 8.51 & 2.24 & 0.31 & 2.14 & 0.352 & 0.055 & 0.194 & 0.042 \\
\hline DP11059 & 9 & 2 & 1.29 & 250.79 & 0.77 & 4.86 & 24.9 & 7.55 & 2.22 & 0.30 & 1.92 & 0.346 & 0.044 & 0.218 & 0.042 \\
\hline DP11060 & 9 & 3 & 0.25 & 251.25 & 0.99 & 4.83 & 24.2 & 7.52 & 2.17 & 0.33 & 1.85 & 0.330 & 0.065 & 0.232 & 0.041 \\
\hline DP11061 & 9 & 3 & 0.73 & 251.73 & 1.06 & 4.77 & 23.2 & 7.24 & 2.11 & 0.27 & 1.80 & 0.320 & 0.057 & 0.212 & 0.017 \\
\hline DP11062 & 9 & 4 & 0.25 & 252.75 & 1.30 & 4.97 & 25.9 & 8.09 & 2.33 & 0.43 & 1.85 & 0.339 & 0.077 & 0.171 & 0.026 \\
\hline DP11063 & 9 & 4 & 0.73 & 253.23 & 1.13 & 4.75 & 24.6 & 7.77 & 2.15 & 0.35 & 1.80 & 0.332 & 0.063 & 0.156 & 0.026 \\
\hline DP11064 & 10 & 1 & 0.11 & 257.61 & 1.35 & 5.03 & 23.6 & 8.18 & 2.27 & 0.30 & 1.81 & 0.349 & 0.061 & 0.113 & 0.006 \\
\hline DP11065 & 10 & 1 & 0.81 & 258.31 & 1.56 & 4.85 & 23.4 & 8.06 & 2.18 & 0.41 & 1.81 & 0.353 & 0.082 & 0.130 & 0.016 \\
\hline DP1 1066 & 10 & 1 & 1.41 & 258.91 & 1.73 & 4.99 & 19.7 & 6.94 & 1.96 & 0.31 & 1.46 & 0.343 & 0.058 & 0.102 & 0.007 \\
\hline DP1 1067 & 10 & 2 & 0.11 & 259.11 & 1.66 & 4.29 & 22.2 & 7.93 & 2.01 & 0.40 & 1.48 & 0.292 & 0.078 & 0.120 & 0.014 \\
\hline DP11068 & 10 & 2 & 0.81 & 259.81 & 2.61 & 4.73 & 22.3 & 8.10 & 2.43 & 0.57 & 1.55 & 0.317 & 0.174 & 0.112 & 0.023 \\
\hline DP11069 & 10 & 2 & 1.41 & 260.41 & 2.35 & 4.77 & 21.4 & 7.81 & 2.25 & 0.38 & 1.67 & 0.343 & 0.107 & 0.103 & 0.000 \\
\hline DP11070 & 10 & 3 & 0.11 & 260.61 & 2.91 & 5.20 & 21.7 & 8.21 & 2.41 & 0.58 & 1.71 & 0.355 & 0.181 & 0.085 & 0.021 \\
\hline DP11071 & 10 & 3 & 0.81 & 261.31 & 2.05 & 4.67 & 21.9 & 7.92 & 2.58 & 0.59 & 1.90 & 0.341 & 0.178 & 0.041 & 0.021 \\
\hline DP11072 & 10 & 3 & 1.31 & 261.81 & 1.62 & 5.47 & 20.1 & 7.08 & 2.07 & 0.76 & 1.72 & 0.493 & 0.229 & 0.044 & 0.025 \\
\hline DP11073 & 10 & 4 & 0.11 & 262.11 & 1.39 & 4.96 & 22.8 & 7.66 & 2.08 & 0.29 & 1.76 & 0.340 & 0.059 & 0.128 & 0.023 \\
\hline DP1 1074 & 10 & 4 & 0.61 & 262.61 & 1.82 & 5.18 & 28.1 & 9.28 & 2.34 & 0.33 & 2.29 & 0.380 & 0.070 & 0.189 & 0.025 \\
\hline DP1 1075 & 10 & 5 & 0.11 & 263.61 & 1.25 & 5.02 & 26.1 & 8.74 & 2.38 & 0.32 & 2.06 & 0.371 & 0.062 & 0.130 & 0.026 \\
\hline DP11076 & 10 & 5 & 0.61 & 264.11 & 0.99 & 4.83 & 23.8 & 7.96 & 2.25 & 0.29 & 1.84 & 0.353 & 0.056 & 0.110 & 0.022 \\
\hline DP1 1077 & 10 & 6 & 0.11 & 265.11 & 1.29 & & & & & 0.32 & 2. & & 0.069 & 0.162 & 0.032 \\
\hline DP11078 & 11 & 1 & 0.17 & 267.17 & 1.85 & 5.07 & 24.1 & 8.60 & 2.79 & 0.55 & 2.12 & 0.392 & 0.186 & 0.044 & 0.026 \\
\hline DP1 1079 & 12 & 1 & 0.21 & 276.71 & 0.11 & 1.16 & 33.9 & 1.08 & 0.47 & 0.68 & 0.42 & 0.085 & 0.248 & 0.003 & 0.023 \\
\hline DP1 1080 & 12 & 1 & 0.37 & 276.87 & 0.00 & 1.40 & 33.3 & 1.15 & 0.56 & 0.49 & 0.45 & 0.091 & 0.186 & 0.000 & 0.016 \\
\hline DP1 1081 & 13 & 1 & 0.04 & 286.04 & 0.00 & 0.32 & 31.3 & 0.21 & 0.17 & 0.23 & 0.04 & 0.091 & 0.117 & 0.000 & 0.016 \\
\hline DP11082 & 14 & 1 & 0.22 & 295.72 & 0.34 & 1.71 & 33.5 & 0.77 & 0.42 & 0.28 & 0.33 & 0.059 & 0.117 & 0.029 & 0.008 \\
\hline DP11083 & 15 & 1 & 0.34 & 305.34 & 0.51 & 2.35 & 33.5 & 0.19 & 0.17 & 0.25 & 0.07 & 0.040 & 0.086 & 0.035 & 0.006 \\
\hline DP1 1084 & 15 & 1 & 0.95 & 305.95 & 0.37 & 2.11 & & 0.27 & 0.22 & 0.21 & 0.07 & 0.031 & 0.080 & 0.024 & 0.005 \\
\hline DP1 1085 & 16 & 1 & 0.23 & 314.72 & 0.68 & 2.58 & 34.2 & 0.61 & 0.42 & 0.53 & 0.30 & 0.051 & 0.182 & 0.119 & 0.022 \\
\hline DP11086 & 17 & 1 & 0.12 & 324.12 & 0.15 & 1.67 & 33.3 & 0.19 & 0.24 & 0.19 & 0.09 & 0.026 & 0.089 & 0.095 & 0.041 \\
\hline DP11087 & 17 & 1 & 0.30 & 324.30 & 0.00 & 1.76 & 34.0 & 0.22 & 0.25 & 0.10 & 0.11 & 0.026 & 0.051 & 0.000 & 0.000 \\
\hline DP1 1088 & 17 & 1 & 1.10 & 325.10 & 0.31 & 2.24 & 32.6 & 0.14 & 0.11 & 0.04 & 0.03 & 0.037 & 0.031 & 0.000 & 0.000 \\
\hline DP1 1089 & 18 & 1 & 0.19 & 333.69 & 0.44 & 1.93 & 29.9 & 0.13 & 0.10 & 0.05 & 0.02 & 0.020 & 0.033 & 0.000 & 0.000 \\
\hline
\end{tabular}

on all elemental abundance axes are positive). These rotated axes can then be converted to actual elemental compositions and can be treated as physical end members of the population. This technique was developed by Leinen and Pisias (1984). We have used their algorithm to analyze the Leg 86 data.

Table 4 shows the compositions of the reference (rotated) axes for the combined Site 579 and 580 data sets. Figure 6 shows how these factors load on to the downhole samples.

Factor 1 is primarily terrigenous detritus with some biogenic opal. The Site 580 samples are slightly depleted in this factor and are markedly more variable than the Site 579 samples (Fig. 6). In contrast, Factor 3, which is dominated by biogenic opal and $\mathrm{Mn}$, is much more abundant in the Site 580 samples from the more productive northern site. Factor 2 is a mixture of authigenic iron sulfide and terrigenous detritus. Its greater abundance at Site 580 is consistent with a greater influx of organic carbon, which has served as the energy source for sulfate-reducing bacteria at this site.

If sulfur is excluded from the factor analysis, three simpler end members emerge (Table 5, Fig. 7). Factor 1 is again dominated by terrigenous debris, in this case with a smaller addition of biogenic opal. The downhole patterns at Sites 579 and 580 are very similar, with the abundance of factor 1 roughly doubling from the oldest (Pliocene) to youngest (Quaternary) samples. The opalrich Factor 3 shows almost the inverse pattern, with a marked decrease from older to younger samples. At Site 580 , the transition is continuous, with a lot of variability throughout the section. In contrast, the abundance of 


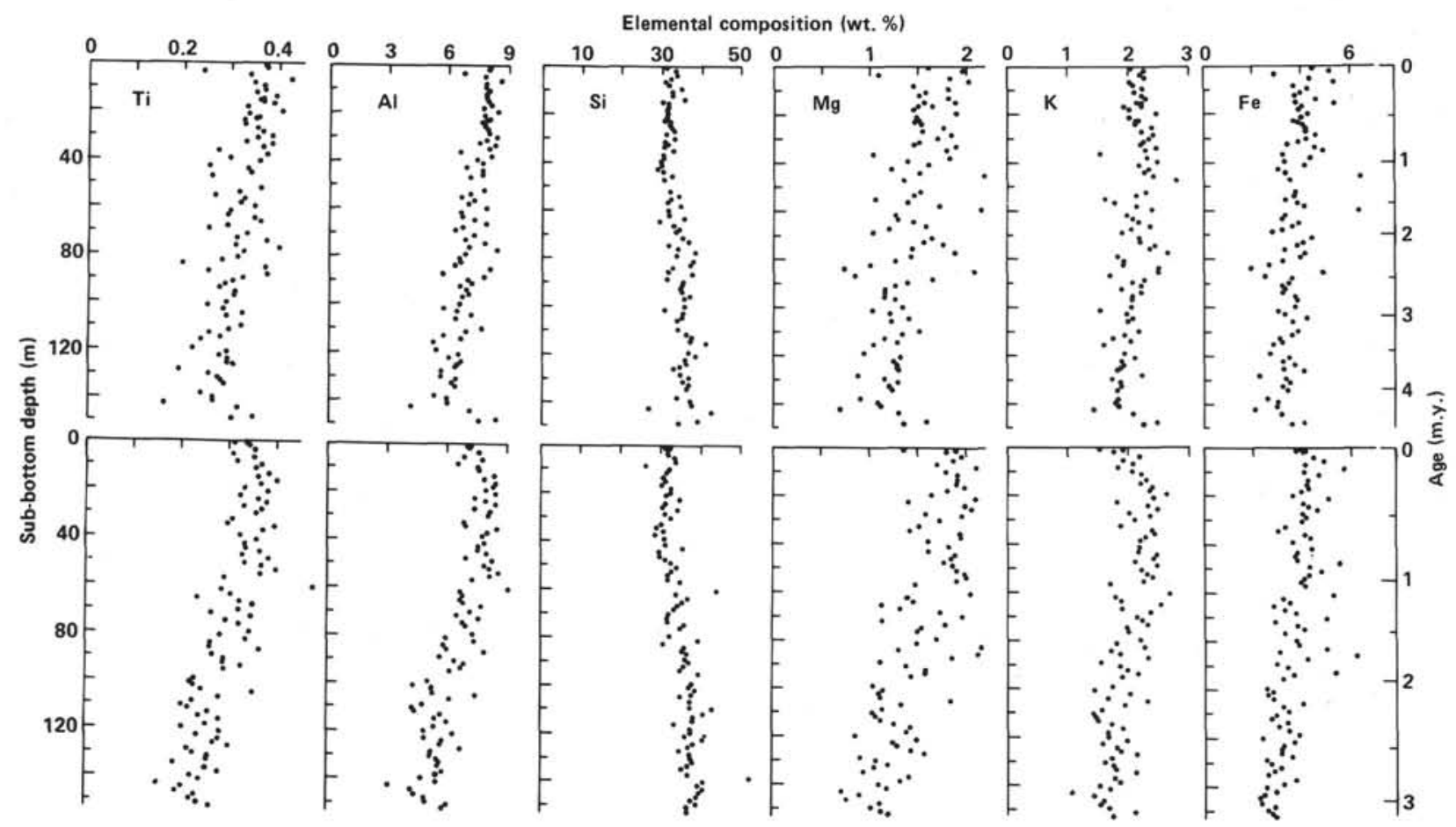

Figure 2. Downhole profiles of elemental abundances in samples from Sites 579 (upper) and 580 (lower). Analyses by X-ray fluorescence, salt corrected. The data are given in Tables 1 and 2 .

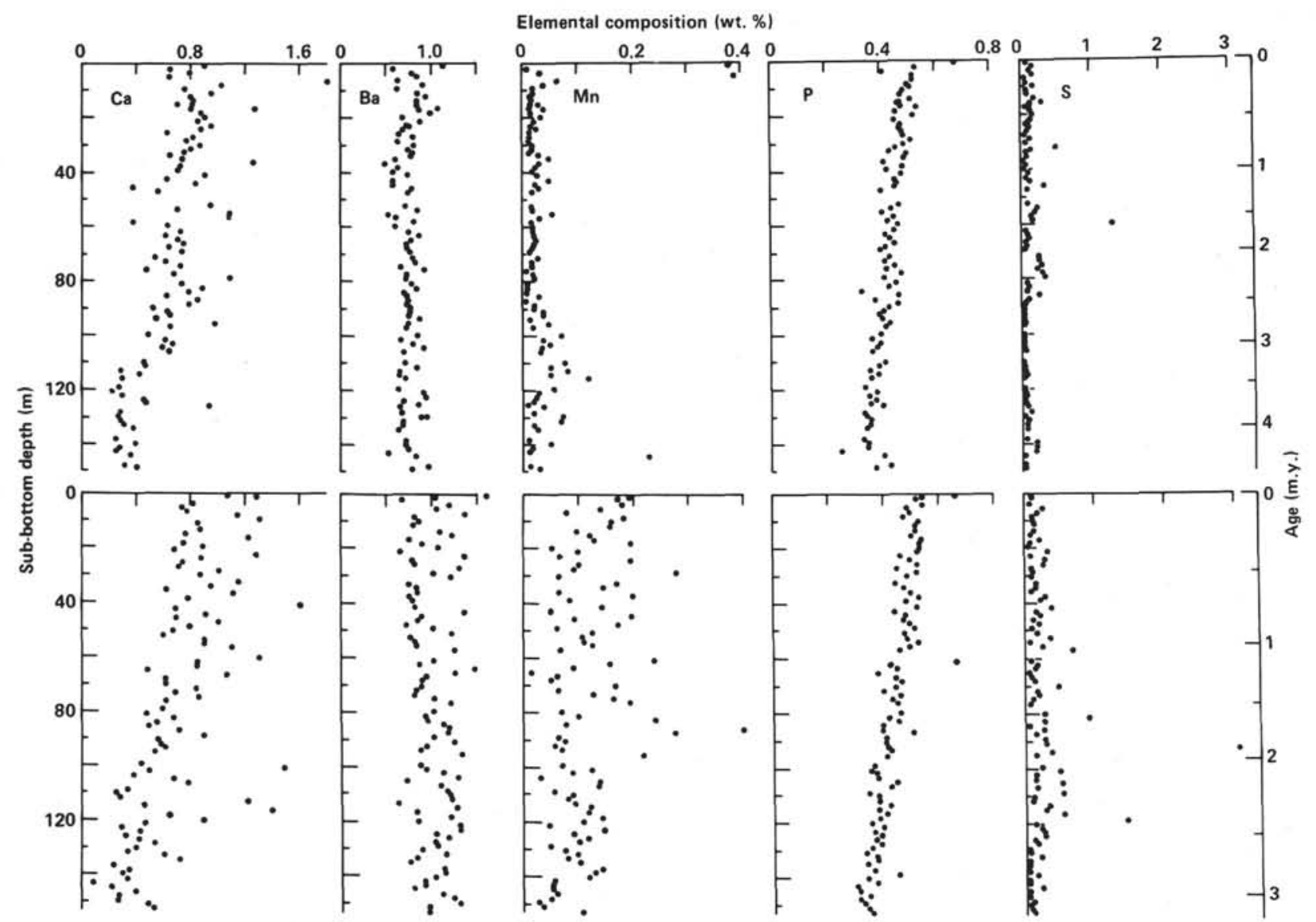

Figure 3. Downhole profiles of minor element abundances in samples from Sites 579 (upper) and 580 (lower). Analyses by X-ray fluorescence, salt corrected. The data are given in Tables 1 and 2 . 


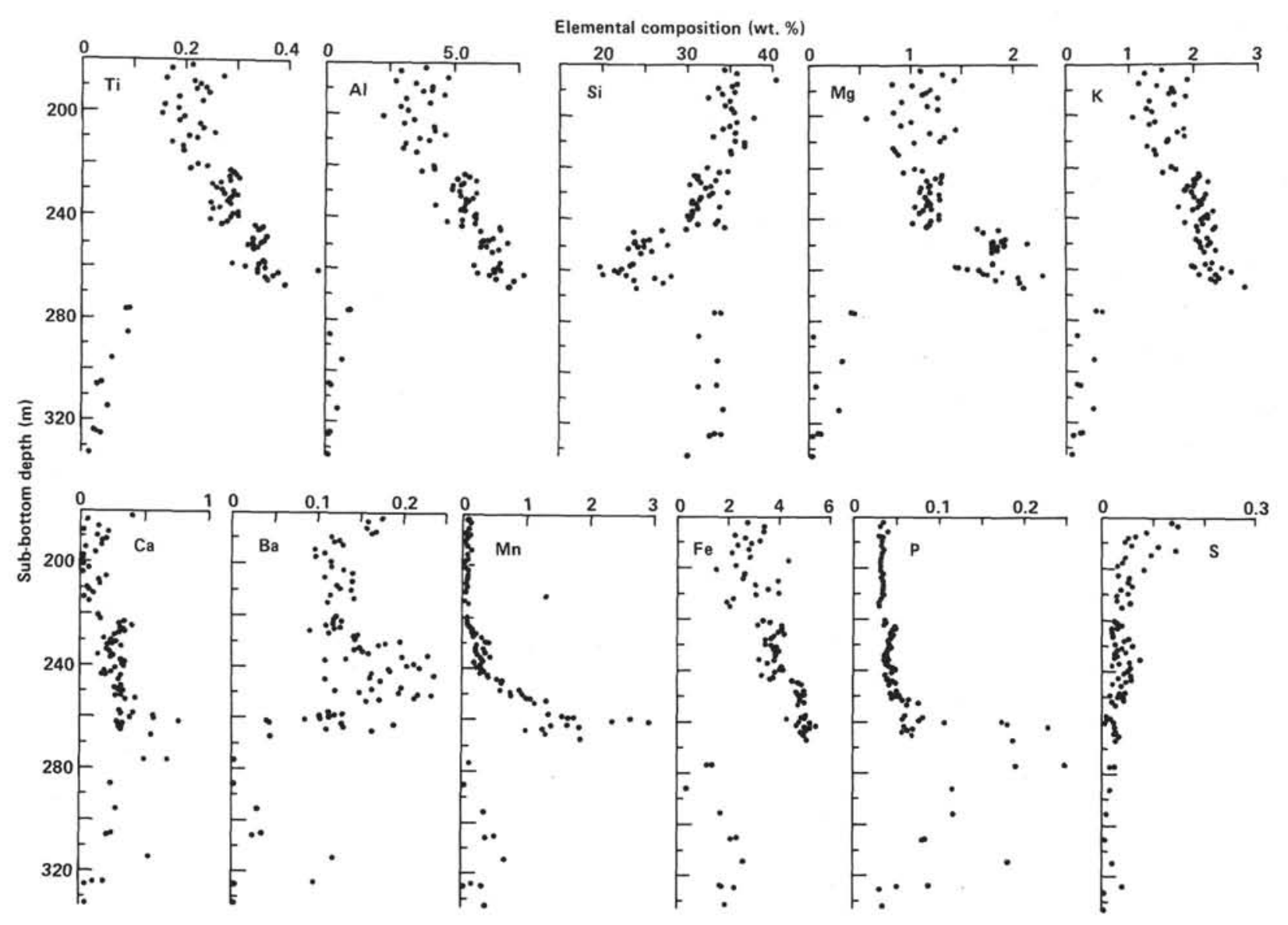

Figure 4. Downhole profiles of elemental abundances in samples from Site 581. Analyses by X-ray fluorescence, salt corrected. The data are given in Table 3.

Factor 3 at Site 579 decreases abruptly near $110 \mathrm{~m}$, corresponding to an age of about 3.2 m.y. Factor 2 , which consists of terrigenous detritus enriched in authigenic (?) iron and manganese, is more abundant at Site 580 than at Site 579, but shows little variation downhole.

The results of factor analysis of the Site 581 samples are shown in Figure 8. The Q-mode analysis (Table 6) distinguishes detrital, Mn-rich, and biogenic factors (Factors 1,2 , and 3 , respectively). The detrital factor decreases continuously in importance from the basal clays to the cherts below $276 \mathrm{~m}$. The other two factors show sharp discontinuities at this boundary.

The rotated factors (Table 7), in contrast, vary much more smoothly across the clay/chert boundary. The detrital-rich Factor 1 decreases to very low values in the chert, with only three elevated values near $325 \mathrm{~m}$. Factor 3 , dominated by opal and minor fish debris, is uniformly high in the chert, decreases to a minimum in the dark brown clays (245-276 m), and increases slightly in the less oxidized clays. Factors 2, which is dominated by an authigenic ferromanganese component with subordinate fish debris and detritus, is concentrated in the dark brown clays $(245-276 \mathrm{~m})$. Lower but still significant levels are evident in the light brown clays (224-245 m) and chert (below $276 \mathrm{~m}$ ). Only the reduced (gray green) clays above $224 \mathrm{~m}$ are depleted in this factor.

\section{DISCUSSION AND CONCLUSIONS}

The elemental composition of sediments at Sites 579, 580 , and 581 is dominated by contributions from terrigenous detritus and opal-rich biogenic debris. Redox-driven diagenetic reactions (mobilization and redistribution of manganese and precipitation of iron sulfide) and the deposition of authigenic ferromanganese oxyhydroxides in mid-Tertiary dark brown clays at Site 581 modify the abundances of some elements.

From a geochemical perspective, Sites 579 and 580 are almost indistinguishable. A slight enrichment of the biogenically associated elements $\mathrm{Si}, \mathrm{Ca}, \mathrm{Ba}$, and $\mathrm{P}$ at Site 580 , which lies beneath more biologically productive waters than Site 579, is the major difference. At both sites, the ratio of detrital to biogenic debris increases uphole from the late Pliocene to the late Quaternary portions of the profiles. It seems unlikely that this trend resulted from a decrease in biogenic input, given the northward drift of the sites and more vigorous oceanic circulation during the Quaternary in this region. Rather, the trend probably reflects the accelerated eolian deposition of continental debris from loess deposits in Asia that accompanied the development of Northern Hemisphere glaciation and that has been recognized elsewhere in the North Pacific (Heath, 1969; Leinen and Heath, 1981). 


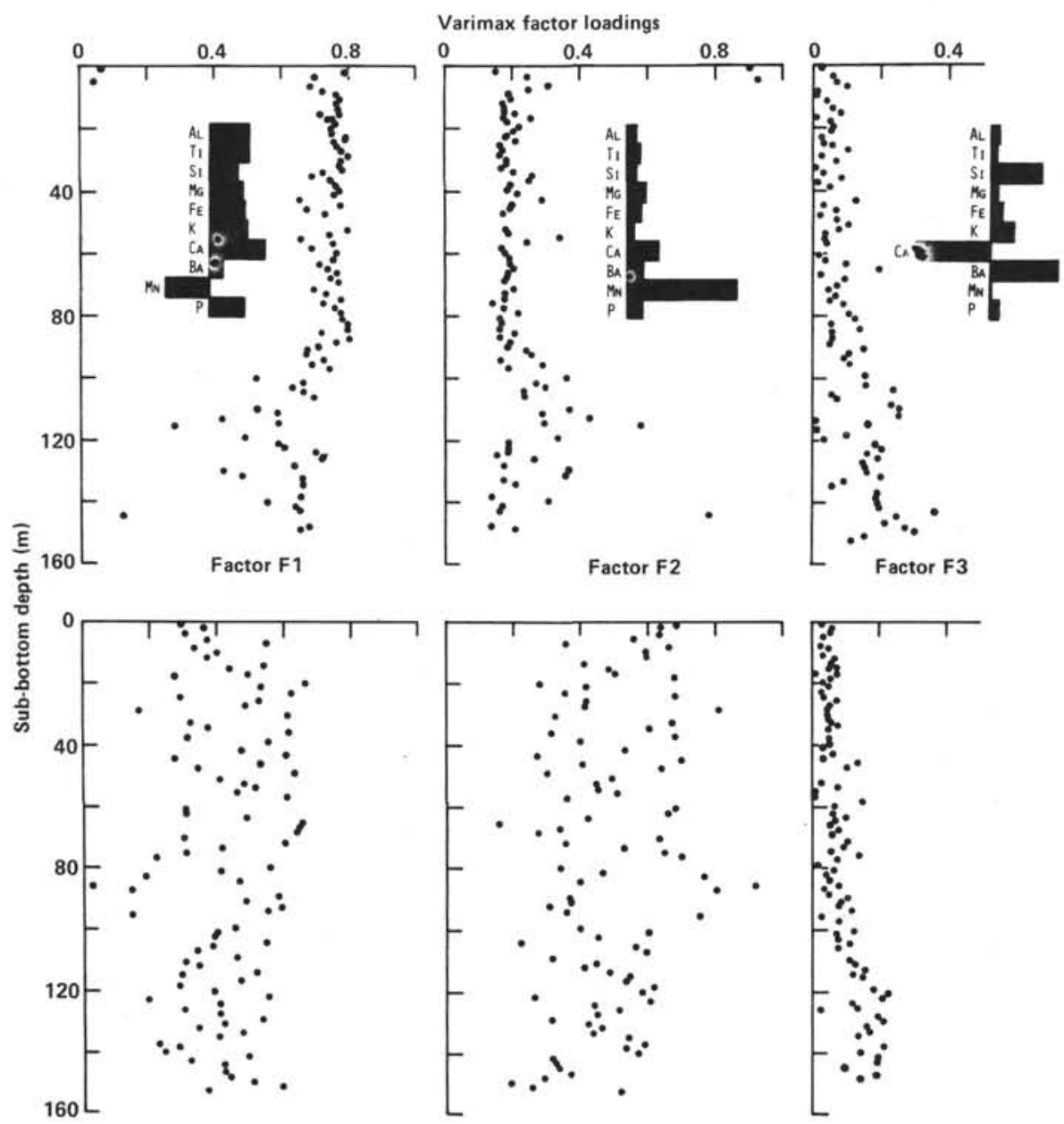

Figure 5. Downhole profiles of loadings of Q-mode varimax factors on samples from Sites 579 (upper) and 580 (lower). Histograms show contributions of elements to each factor.

Table 4. Composition (wt. \%) of the rotated Q-mode factors for Sites 579 and 580 plotted in Figure 6.

\begin{tabular}{lccc}
\hline Element & Factor 1 & Factor 2 & Factor 3 \\
\hline $\mathrm{Ti}$ & 0.31 & 0.35 & 0.25 \\
$\mathrm{Al}$ & 7.09 & 6.53 & 5.18 \\
$\mathrm{Si}$ & 32.4 & 25.3 & 34.3 \\
$\mathrm{Mg}$ & 1.35 & 2.65 & 1.62 \\
$\mathrm{~K}$ & 2.14 & 1.83 & 1.53 \\
$\mathrm{Fe}$ & 3.51 & 6.36 & 3.48 \\
$\mathrm{Ca}$ & 0.65 & 1.39 & 0.62 \\
$\mathrm{Ba}$ & 0.08 & 0.07 & 0.14 \\
$\mathrm{Mn}$ & 0.00 & 0.005 & 0.48 \\
$\mathrm{P}$ & 0.04 & 0.05 & 0.04 \\
$\mathrm{~S}$ & 0.03 & 3.08 & 0.00 \\
\end{tabular}

A feature deserving of additional study is the enhanced deposition of Mn at Site 580 relative to Site 579 . We suspect that the excess Mn is preserved as a carbonate, but the depositional conditions responsible for such preservation have not been identified.

The record at Site 581 captures the basal part of the sequence cored at Sites 579 and 580 (reduced, opal-bearing detrital clays), as well as oxidized detrital-rich clays and highly oxidized pelagic clays rich in authigenic fer- romanganese oxyhydroxides. This section overlies porcellaneous chert.

The oxidized clays are reminiscent of "red clay" sequences elsewhere in the North Pacific (see Site 576 and 578 chapters, this volume; Leinen, 1979). Both P and $\mathrm{Ba}$ are enriched in the darkest brown clays, reflecting the concentration of the most refractory biogenic debris (phosphatic fish fragments and barite) in the least rapidly accumulating sediments.

The porcellaneous cherts at Site 581 contain some surprises. They contain about the same concentration of sili$\mathrm{ca}$ as the oxidized detrital clays and markedly less than the biosiliceous clays at Site 580 . The relatively high Mn contents and red to yellow colors also point to fairly slow deposition in a well-oxygenated environment. The high $\mathrm{P}$ contents of the cherts, particularly the youngest ones, also is suggestive of slow deposition (i.e., greater relative abundance of phosphatic fish debris). The possibility that precipitation of the chert layers concentrates fish debris (perhaps by physically displacing the finer clay particles) cannot be excluded, however.

Overall, the chemical data are consistent with the paleoceanographic interpretations derived from lithologic and paleontologic descriptions of the sections at Sites 

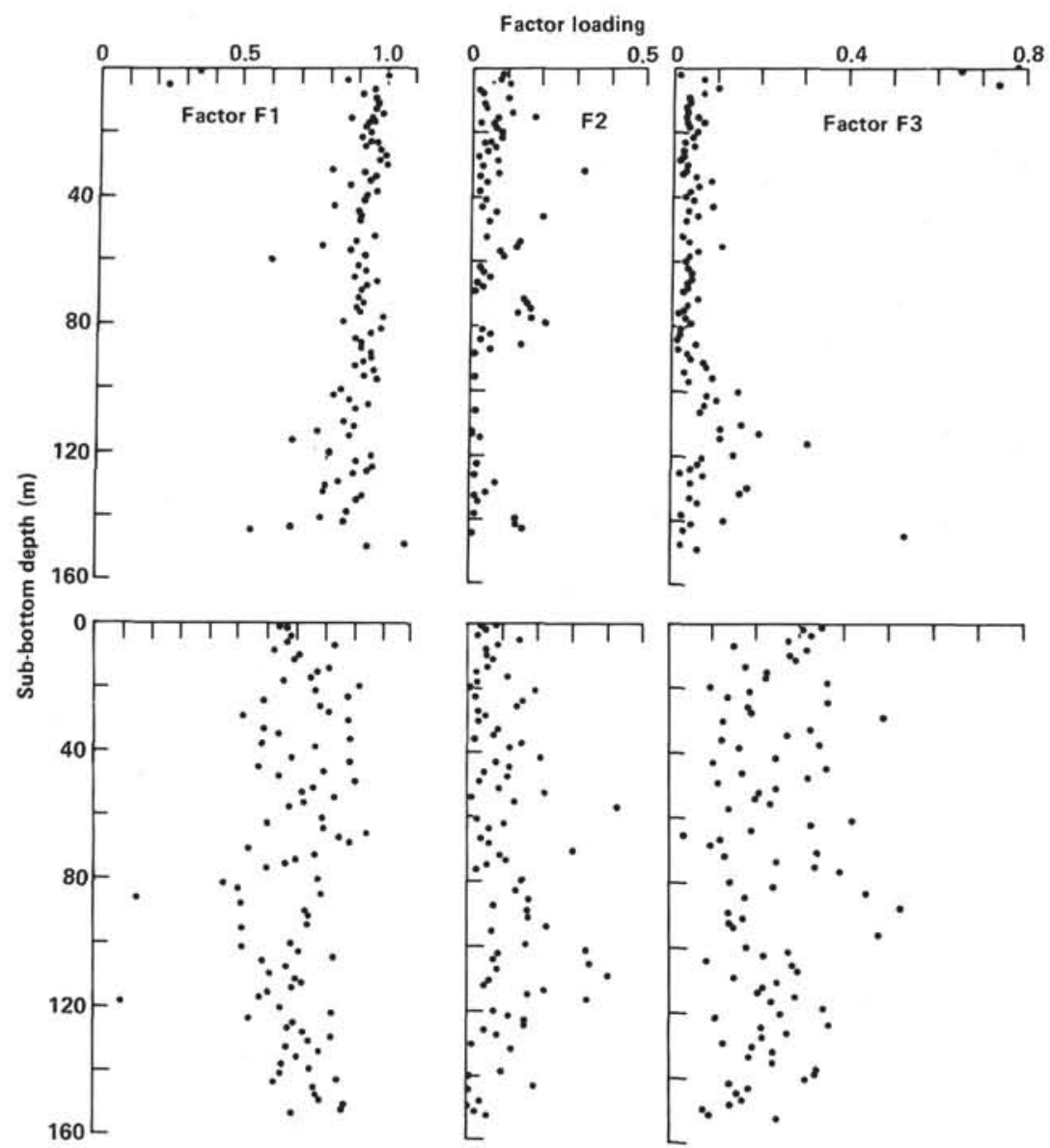

Figure 6. Downhole profiles of loadings of rotated varimax factors (see text for explanation) on samples from Sites 579 (upper) and 580 (lower). Compositions of rotated factors are listed in Table 4. All elements, including sulfur, are included.

Table 5. Composition (wt.\%) of the rotated Q-mode factors (S omitted) for Sites 579 and 580 plotted in Figure 7.

\begin{tabular}{lccc}
\hline Element & Factor 1 & Factor 2 & Factor 3 \\
\hline $\mathrm{Ti}$ & 0.34 & 0.35 & 0.23 \\
$\mathrm{Al}$ & 7.75 & 6.57 & 5.16 \\
$\mathrm{Si}$ & 30.7 & 28.2 & 36.0 \\
$\mathrm{Mg}$ & 1.52 & 2.63 & 1.10 \\
$\mathrm{~K}$ & 2.22 & 1.38 & 1.81 \\
$\mathrm{Fe}$ & 3.91 & 5.15 & 2.90 \\
$\mathrm{Ca}$ & 0.94 & 2.10 & 0.00 \\
$\mathrm{Ba}$ & 0.07 & 0.14 & 0.11 \\
$\mathrm{Mn}$ & 0.00 & 0.90 & 0.06 \\
$\mathrm{P}$ & 0.05 & 0.06 & 0.03 \\
\hline
\end{tabular}

579,580 , and 581 . The sets of closely spaced analyses presented here yield a measure of both the general trends and variability of deposition at the three sites. This data set should also be useful for future studies of the comparative geochemistry of North Pacific pelagic clays.

\section{ACKNOWLEDGMENTS}

We are grateful to the members of the scientific party of Leg 86 for discussions concerning the deposition of western North Pacific pelagic sediments. We also acknowledge the assistance of Ivan Pavlov and
Greg Campi in preparing and analyzing samples, and helping to plot the results. This manuscript was improved by the reviews of Miriam Kastner and Mitchell Lyle.

\section{REFERENCES}

Chase, T. E., 1975. Topography of the oceans. Univ. Calif. San Diego, Scripps Inst. Oceanog., Inst. Mar. Resources Tech. Rept., Ser. TR57. Coleman, M., Fleet., A., and Donson, P., 1982. Preliminary studies of manganese-rich carbonate nodules from Leg 68, Site 503, eastern equatorial Pacific. In Prell, W. L., Gardner, J. V., et al., Init. Repts. DSDP, 68: Washington (U.S. Govt. Printing Office), 481489.

Criss, J. W., Birks, L. S., and Gilfrich, J. V., 1978. A versatile x-ray analysis program combining fundamental parameters and empirical coefficients. Anal. Chem., 50:33.

Dymond, J., 1981. The geochemistry of Nazca Plate surface sediments: an evaluation of hydrothermal, biogenic, detrital and hydrogenous sources. Mem. Geol. Soc. Am., 154:133-173.

Froelich, P., Klinkhammer, G., Bender, M., Luedtke, N., Heath, R., Cullen, D., Dauphin, P., Hammond, D., Hartman, B., and Maynard, V., 1979. Early oxidation of organic matter in pelagic sediments of the eastern equatorial Atlantic: suboxic diagenesis. Geochim. Cosmochim. Acta, 43:1075-1090.

Heath, G. R., 1969. Mineralogy of Cenozoic deep-sea sediments from the equatorial Pacific Ocean. Geol. Soc. Am. Bull., 80:1997-2018.

Klovan, J. E., and Imbrie, J., 1971. An algorithm and FORTRAN IV program for large scale Q-mode factor analysis. J. Int. Assoc. Math. Geol., 3:61-77.

Leinen, M., 1979. Paleochemical signatures in Cenozoic Pacific sediments [Ph.D. dissert.]. University of Rhode Island, Narragansett. 
Leinen, M., and Heath, G. R., 1981. Sedimentary indicators of atmospheric activity in the northern hemisphere during the Cenozoic. Palaeogeogr., Palaeoclimatol., Palaeoecol., 36:1-21.

Leinen, M., and Pisias, N. G., 1984. An objective technique for determining end-member compositions and for partitioning sediments according to their sources. Geochim. Cosmochim. Acta, 48:47-63.

Leinen, M., and Stakes, D., 1979. Metal accumulation rates in the central equatorial Pacific during Cenozoic time. Geol. Soc. Am. Bull., 90:357-375.
Lyle, M. W., 1983. The brown-green color transition in marine sediments: a marker of the Fe(III)-Fe(II) redox boundary. Limnol. Oceanogr., 28:1026-1033.

Pederson, T. F., and Price, N. B., 1982. The geochemistry of manganese carbonate in Panama Basin sediments. Geochim. Cosmochim. Acta, 46:59-68.

Date of Initial Receipt: 1 May 1984

Date of Acceptance: 25 September 1984

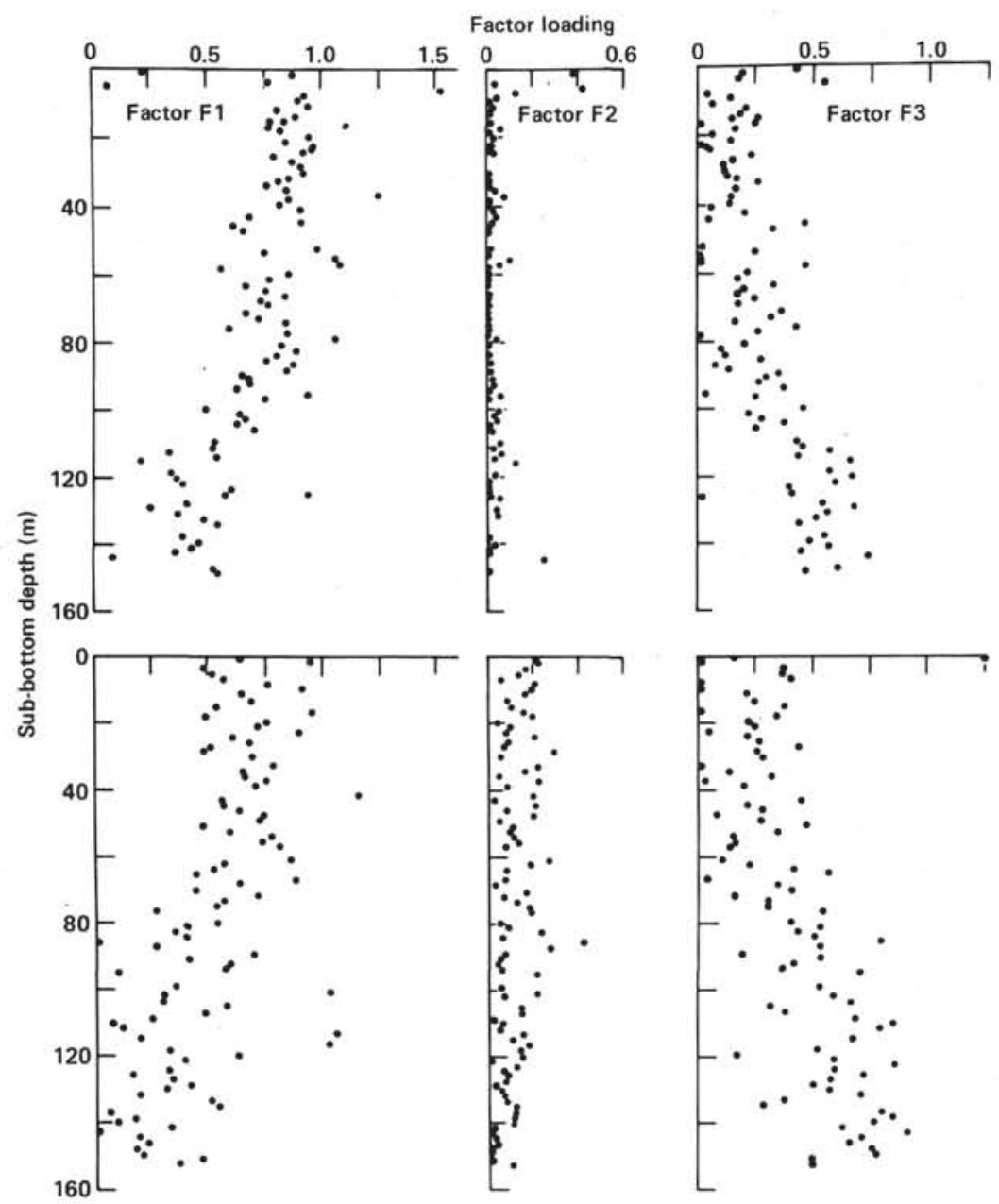

Figure 7. As Figure 6, but for factors that do not include sulfur (see Table 5 for factor compositions).

Table 6. Elemental contributions (factor scores) to Q-mode varimax factors for Site 581 analyses (Fig. 8).

\begin{tabular}{lrrr}
\hline Element & Factor 1 & Factor 2 & Factor 3 \\
\hline $\mathrm{Ti}$ & 1.04 & 0.54 & -0.20 \\
$\mathrm{Al}$ & 1.05 & 0.68 & -0.45 \\
$\mathrm{Si}$ & 1.27 & -0.01 & 1.37 \\
$\mathrm{Mg}$ & 1.02 & 0.66 & -0.36 \\
$\mathrm{~K}$ & 1.17 & 0.43 & -0.27 \\
$\mathrm{Ca}$ & 0.16 & 0.70 & 1.19 \\
$\mathrm{Ba}$ & 1.49 & 0.10 & -0.33 \\
$\mathrm{Mn}$ & -0.87 & 2.72 & 0.02 \\
$\mathrm{Fe}$ & 0.88 & 0.51 & 0.23 \\
$\mathrm{P}$ & -0.11 & 0.26 & 2.48 \\
\hline
\end{tabular}

Table 7. Composition (wt.\%) of the rotated Q-mode factors for Site 581 plotted in Figure 8.

\begin{tabular}{lccc}
\hline Element & Factor 1 & Factor 2 & Factor 3 \\
\hline $\mathrm{Ti}$ & 0.23 & 0.01 & 0.04 \\
$\mathrm{Al}$ & 5.15 & 15.0 & 0.00 \\
$\mathrm{Si}$ & 32.4 & 14.6 & 41.2 \\
$\mathrm{Mg}$ & 1.12 & 3.24 & 0.23 \\
$\mathrm{~K}$ & 1.71 & 3.76 & 0.23 \\
$\mathrm{Ca}$ & 0.13 & 0.73 & 0.32 \\
$\mathrm{Ba}$ & 0.15 & 0.19 & 0.01 \\
$\mathrm{Mn}$ & 0.01 & 3.72 & 0.19 \\
$\mathrm{Fe}$ & 3.07 & 8.49 & 1.80 \\
$\mathrm{P}$ & 0.02 & 0.11 & 0.13 \\
\hline
\end{tabular}




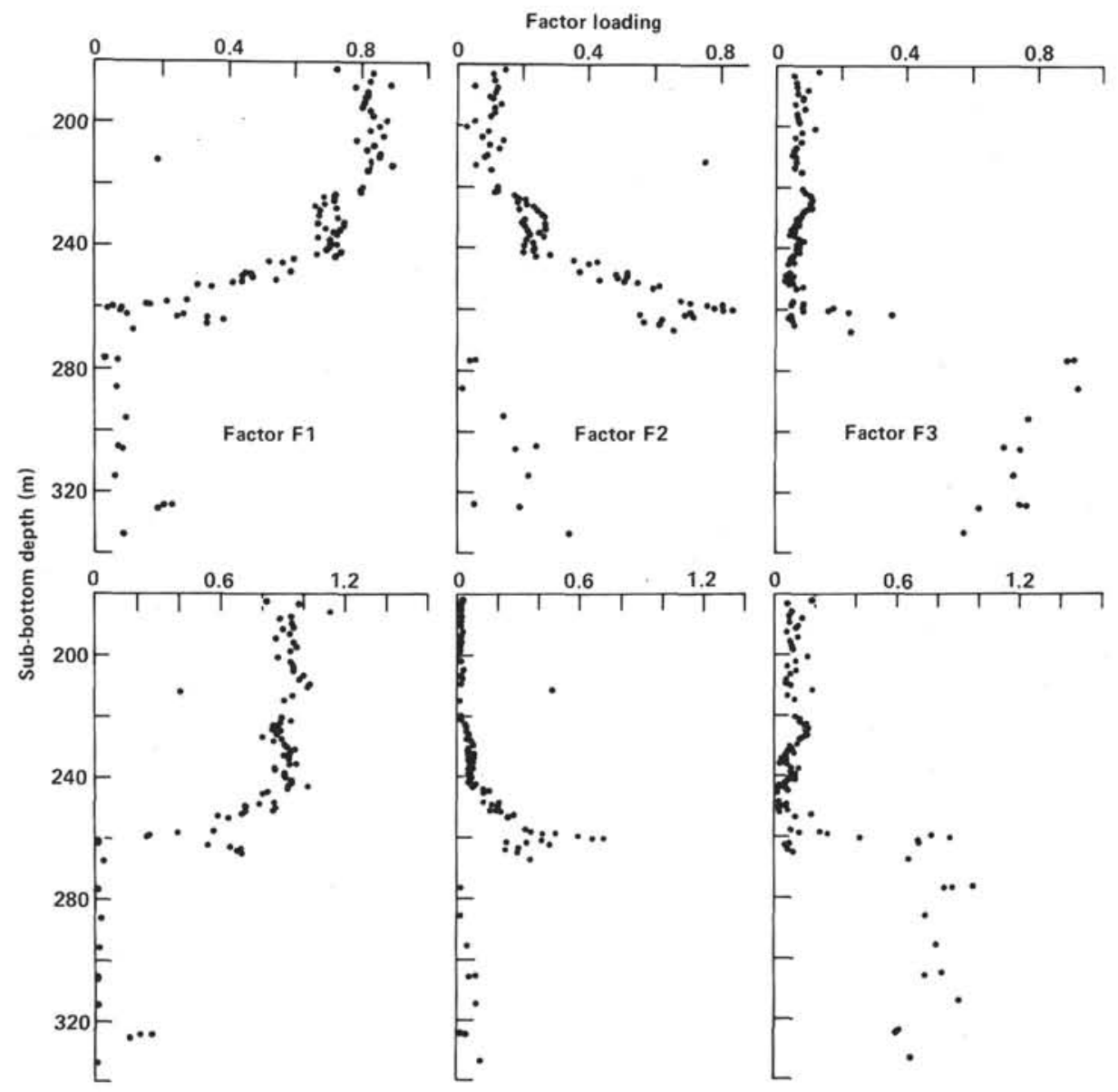

Figure 8. Downhole profiles of loadings of Q-mode varimax factors (upper), and rotated varimax factors (lower) on samples from Site 581. Elemental contributions to Q-mode factors are listed in Table 6. Compositions of rotated factors are listed in Table 7. 